\title{
Zero-phase Velocity Tracking of Vibratory Systems with Actuation Constraints
}

\author{
Luigi Biagiotti ${ }^{\mathrm{a}, *}$, Claudio Melchiorri ${ }^{\mathrm{b}}$ \\ ${ }^{a}$ Department of Engineering "Enzo Ferrari", University of Modena and Reggio \\ Emilia, Via Pietro Vivarelli 10, 41100 Modena, Italy \\ ${ }^{b}$ Department of Electrical, Electronic and Information Engineering "Guglielmo \\ Marconi", University of Bologna, Viale Risorgimento 2, 40136 Bologna, Italy
}

\begin{abstract}
In this paper, a novel filter for precise tracking of constant velocity signals is presented, which allows the reduction of residual vibrations along with the compliance with kinematic constraints that affect the actuation system. A technique achieving both these two objectives at the same time is the main contribution of the work. The filter is based on a cascade of smoothers, i.e. dynamic filters that act on the input signal by increasing its continuity level. Unfortunately, when applied to a generic input composed by ramp (and step) functions, the filter introduces a phase delay not acceptable in many applications where moving parts have to be mated, such as high-speed automatic machines. In order to guarantee a perfect synchronization between the original and the filtered reference signal, once the transient is terminated, a proper compensation scheme has been designed. Moreover, the expressions of the smoothers parameters which assure vibration cancellation at specific
\end{abstract}

\footnotetext{
*Corresponding author. Tel.:+390592056315; fax:+390592056329.

Email addresses: luigi.biagiotti@unimore.it (Luigi Biagiotti), claudio.melchiorri@unibo.it (Claudio Melchiorri)
} 
frequencies and compliance with given bounds on velocity and acceleration have been analytically deduced. By means of an extensive experimental activity, the effectiveness of the proposed approach has been demonstrated, by comparing its performances with the results of well established approaches for vibrations suppression or signal derivatives limitation.

Keywords: Feed-forward control, Smoothers, Residual vibration, Input shaping, Kinematic constraints, Trajectory planning

\section{Introduction}

The growing interest for online trajectory planning has led to the development of a number of filters that, on the basis of rough reference signals, like e.g. steps and ramps, produce smooth functions that meet given specifications, such as constraints on velocity, acceleration and higher derivatives, or the reduction of residual vibrations caused by vibratory modes of the plant. Unfortunately, the proposed solutions are usually focussed on specific issues and do not cope with motion problems from a global perspective. In this respect, a wide literature exists on the online generation of minimum-time trajectories compliant with kinematic constraints (see for instance Lo Bianco et al. (2000); Kröger et al. (2006); Nguyen et al. (2007); Haschke et al. (2008); Gerelli and Bianco (2010); Kröger and Wahl (2010); Ezair et al.; Biagiotti and Zanasi (2010); Thirachai et al. (2010); Guarino Lo Bianco and Ghilardelli (2014); Nakabayashi et al. (2017)). In particular, the approaches proposed by Lo Bianco et al. (2000); Gerelli and Bianco (2010); Biagiotti and Zanasi (2010); Thirachai et al. (2010); Guarino Lo Bianco and Ghilardelli (2014); Nakabayashi et al. (2017) can be classified as signal derivatives limiters since 
the output signal is equal to the input as its derivatives comply with the given bounds, otherwise the output signal tends toward the current input value with the maximum allowed speed, acceleration and even jerk depending on the order of the filter. When these systems are fed with a ramp signal, which is discontinuous in velocity, the filtered output reaches the ramp in minimum time by guaranteeing the continuity of velocity and/or acceleration according to a standard multi-segment polynomial profile and then it exactly tracks the input without phase delay. In this case, the problem of vibration suppression is not explicitly addressed even if one of the main reasons for increasing the smoothness of the motion profiles, and in particular for imposing a limited jerk as in Meckl and Arestides (1998); Béarée (2014), is indeed the reduction of the vibrations in resonant plants. On the other hand, the scientific literature related to the suppression of the residual vibration by feed-forward methods is rather wide (see Singer et al. (1999)), including input shaping (Singer and Seering (1990); Singhose et al. (1995); Singh (2004)) and smoothing techniques (Xie et al. (2013b); Biagiotti et al. (2016)) or mixed methods (Pilbauer et al. (2017); Vyhlídal and Hromvcík (2015)), dynamic inversion (Piazzi and Visioli (2000)), etc. However, in the majority of cases these feed-forward algorithms do not consider the kinematic constraints imposed by the task or the actuator, since it is generally supposed that these filtering methods are applied to smooth reference signals that already meet the given constraints. Some attempts of integrating smooth trajectory generation with derivatives limitation and vibrations cancellation have been proposed in the literature, see e.g. Muenchhof and Singh (2003); Biagiotti and Melchiorri (2012); Besset and Béarée (2017). In general, 


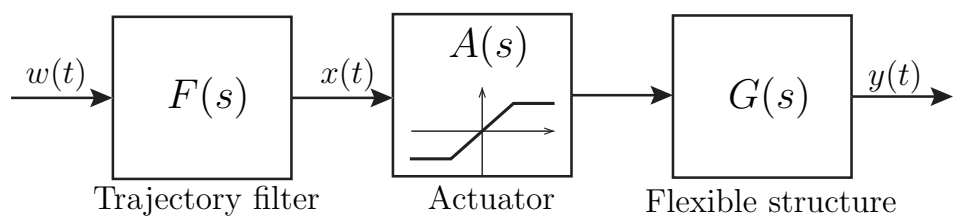

Figure 1: Classical feed-forward structure of a motion system with a vibratory plant.

the application of these methods is mainly limited to rest-to-rest maneuvers, while velocity tracking problems have been tackled only marginally: in Croft and Devasia (1999) an inversion-based approach that compensates for the structural vibrations in the speed control of a scanning tunneling microscopy is proposed, in Masterson et al. (1998) input shaping is used to reduce vibrations in constant-velocity motion during scanning operations too. The main drawback of the feed-forward algorithms applied to constant velocity motions, i.e. ramp functions, is the phase delay that they cause between the reference input and the filtered output. When this delay is a critical issue the feed-forward controller must include a proper compensation. In Kamel et al. (2008) and Peng et al. (2015), schemes based on input shaping techniques have been proposed for zero-phase velocity tracking with residual vibration cancellation.

Inspired by the latter paper, published in this Journal, we propose a novel technique that combines this goal with the compliance with kinematic bounds on velocity and acceleration that may affect the actuation system. Therefore, the scheme of Fig. 1 has been taken into account, where besides the vibratory system also the actuator is considered in the design of the trajectory filter. The key point of the proposed solution is represented by the rectangular and exponential smoothers, that we have introduced in Biagiotti 
and Melchiorri (2012); Biagiotti et al. (2016) in order to take into account both kinematic bounds and vibration suppression specifications. However, it is worth noticing that in previously published results, only rest-to-rest motions obtained by smoothing input step signals have been considered, while in this paper the reference input is composed by ramp functions. Accordingly, because of the different input and because of the term for phase delay compensation, it has been necessary to derive new formulae for the choice of the smoothers parameters, which, together with the general idea of combining different goals in this application, are one the main contribution of this work.

The paper is organized as follows: in Sec. 2 the compensator for asymptotic perfect tracking of ramp signals with feed-forward control proposed by Peng et al. (2015) is generalized in order to take into account the effect of any kind of linear feed-forward filter and, in particular, of the smoothers. Then, in Sections 3 and 4 the design of the chain of exponential and rectangular smoothers for vibration suppression and derivatives saturation with ramp input signals is illustrated. Experimental results, reported in Sec. 5, support the effectiveness of the proposed approach. Concluding remarks are finally reported in Sec. 6.

\section{Compensator for asymptotic perfect tracking of ramp signals}

When a ramp signal $r(t)=v \cdot t$ is applied to the linear system described by the transfer function $F(s)$, supposed asymptotically stable, i.e characterized by $n$ poles $p_{i}$ with $\operatorname{Re}\left\{p_{i}\right\}<0$, its response $\hat{r}(t)$ can be obtained by inverse- 


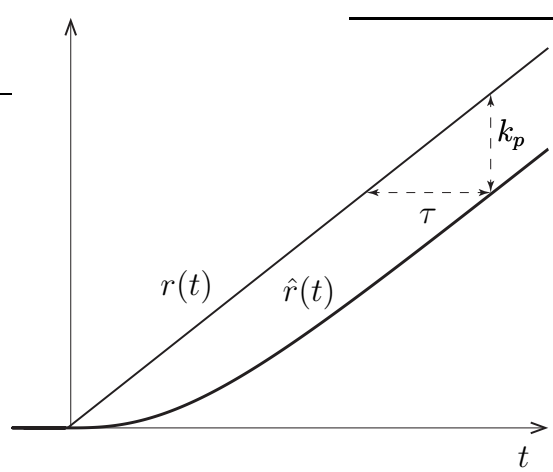

(a)

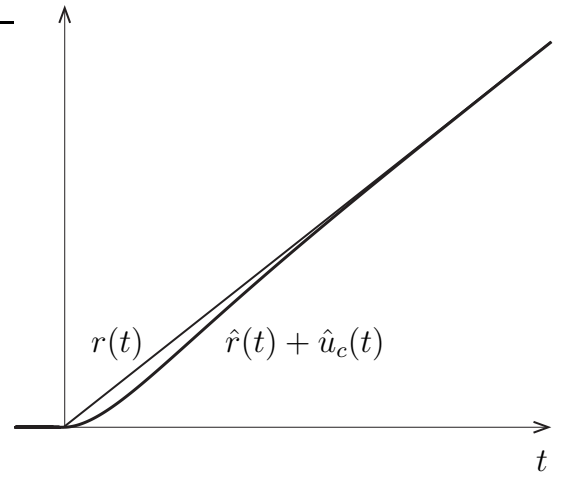

(b)

Figure 2: Ramp signal filtered by a generic dynamic system (a) and filtered ramp with the compensation mechanism reported in Fig. 3 (b).

Laplace transforming

$$
\hat{R}(s)=F(s) \frac{v}{s^{2}}
$$

that can be decomposed as

$$
\hat{R}(s)=\hat{R}_{t}(s)+\frac{k_{v}}{s^{2}}+\frac{k_{p}}{s}
$$

where $\hat{R}_{t}(s)$ contains the terms tied the poles $p_{i}$ of $F(s)$, and the two constants $k_{v}$ and $k_{p}$ are respectively

$$
k_{v}=v \lim _{s \rightarrow 0} F(s), \quad k_{p}=v \lim _{s \rightarrow 0} \frac{d F(s)}{d s} .
$$

By assuming that $F(s)$ has a unity static gain, i.e. $F(0)=1$, it descends that $k_{v}=v$. Finally, the output is

$$
\hat{r}(t)=\hat{r}_{t}(t)+v \cdot t+k_{p}
$$

where the transient term $\hat{r}_{t}=\mathcal{L}^{-1}\left\{\hat{R}_{t}(s)\right\}$ asymptotically vanishes, being the system stable. As shown in Fig. 2(a), the steady-state error between the 


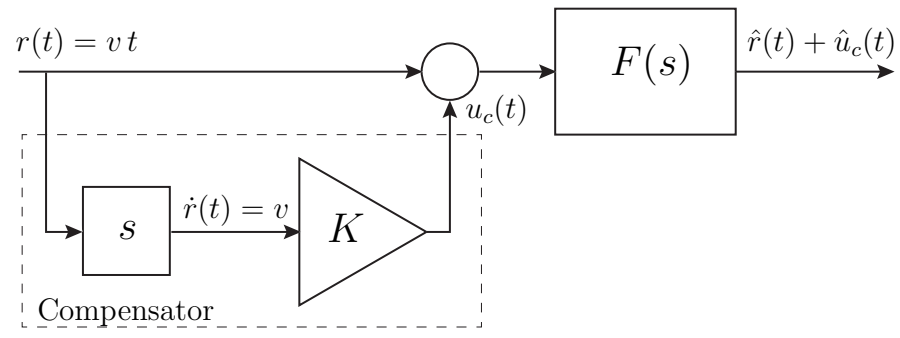

Figure 3: System $F(s)$ with feed-forward compensation for asymptotic perfect tracking of a ramp reference input. The expression of the gain $K$ is reported in (3).

system output $\hat{r}(t)$ and the reference input $r(t)$ is

$$
\bar{e}=\lim _{t \rightarrow \infty}(\hat{r}(t)-r(t))=k_{p}
$$

and therefore it can be compensated with the constant feed-forward action $u_{c}(t)=-k_{p}$, see Fig. 2(b). Note that, being $F(0)=1$, the compensating term can be added either to the input or to the output of $F(s)$, since in any case its asymptotic contribution is $\lim _{t \rightarrow \infty} \hat{u}_{c}(t)=-k_{p}$. If $F(s)$ represents a filter for vibration suppression the former approach is obviously preferable, because also the additional term $u_{c}(t)$ must be filtered. In Fig. 3 the scheme that assures asymptotic perfect tracking of a ramp signal is shown. Note that the compensator is not causal, requiring the derivative of $r(t)$, and accordingly can be realized in this form only if the derivative of the input signal is available. If not, the derivative can be approximated via filtering methods or numerical differentiation like in Peng et al. (2015). The gain of the compensator is

$$
K=-\frac{k_{p}}{v}=-\lim _{s \rightarrow 0} \frac{d F(s)}{d s} .
$$

If the system $F(s)$, that takes into account the filters for reference signal shaping/smoothing as well as the plant, is composed by a cascade of $m$ 
terms, i.e.

$$
F(s)=F_{1}(s) \cdot F_{2}(s) \cdots F_{m}(s)
$$

the gain of the compensator is given by the contribution of each subsystem:

$$
K=\sum_{i=1}^{m} K_{i}
$$

where the parameters $K_{i}$ are computed according to (3). In fact, the product rule for derivatives leads to

$$
K=-\lim _{s \rightarrow 0} \frac{d F(s)}{d s}=-\lim _{s \rightarrow 0} \sum_{i=1}^{m}\left(\frac{d F_{i}(s)}{d s} \prod_{j \neq i} F_{j}(s)\right)=\sum_{i=1}^{m}\left(-\lim _{s \rightarrow 0} \frac{d F_{i}(s)}{d s}\right)
$$

where $\lim _{s \rightarrow 0} \prod_{j \neq i} F_{j}(s)=1$ since the static gain of all the systems $F_{i}(s), i=$ $1, \ldots, m$ is assumed to be unity.

For instance, let us consider the system described in Kamel et al. (2008); Peng et al. (2015), composed by the cascade of a generic Input Shaper

$$
P(s)=\sum_{i=0}^{N-1} A_{i} e^{-s T_{i}}
$$

and a second order system

$$
G(s)=\frac{\omega_{n}^{2}}{s^{2}+2 \delta \omega_{n} s+\omega_{n}^{2}}
$$

describing the plant. In order to compensate for $F(s)=P(s) G(s)$ the gain $K$ must be chosen as

$$
\begin{aligned}
K & =-\lim _{s \rightarrow 0} \frac{d P(s)}{d s}-\lim _{s \rightarrow 0} \frac{d G(s)}{d s} \\
& =\sum_{i=0}^{N-1} A_{i} T_{i}+\frac{2 \delta}{\omega_{n}}
\end{aligned}
$$

which is exactly the result found in Kamel et al. (2008); Peng et al. (2015). 


\section{Scheme based on an exponential smoother for vibration sup- pression}

The suppression of the residual vibration that arises in a resonant system like (4), characterized by a natural frequency $\omega_{n}$ and a damping coefficient $\delta$, can be obtained by means of a so-called exponential smoother (see Xie et al. (2013a); Biagiotti et al. (2016)). The transfer function of the smoother is

$$
F_{e x p}(s)=\frac{\sigma}{e^{\sigma T}-1} \frac{1-e^{\sigma T} e^{-T s}}{s-\sigma}
$$

where $\sigma$ and $T$ are free parameters that can be chosen in order to guarantee the cancellation of the poles of (4) that cause vibration. As shown by Biagiotti et al. (2016), the filter $F_{\text {exp }}(s)$ guarantees the complete residual vibration suppression if

$$
\begin{aligned}
& \sigma=-\delta \omega_{n} \\
& T=k \frac{2 \pi}{\omega_{n} \sqrt{1-\delta^{2}}}=k T_{0} \quad k=1,2, \ldots
\end{aligned}
$$

being $T_{0}=\frac{2 \pi}{\omega_{n} \sqrt{1-\delta^{2}}}$ the period of the oscillation. Besides the different robustness with respect to errors in the estimation of the plant parameters $\delta$ and $\omega_{n}$, aspect that has been fully analyzed in Biagiotti et al. (2016), a noteworthy advantage of smoothers with respect to input shapers, that have been previously used in similar applications, is their capability of increasing the smoothness, i.e. the continuity level, of the filtered signal. In particular, the exponential filter increases by one the continuity level of the input signal. For instance in case of an input composed only by ramp functions, and therefore $\mathcal{C}^{0}$, the filter output will be $\mathcal{C}^{1}$, i.e. with continuous first derivative. Since the exponential filter not only increases the continuity level of the input 


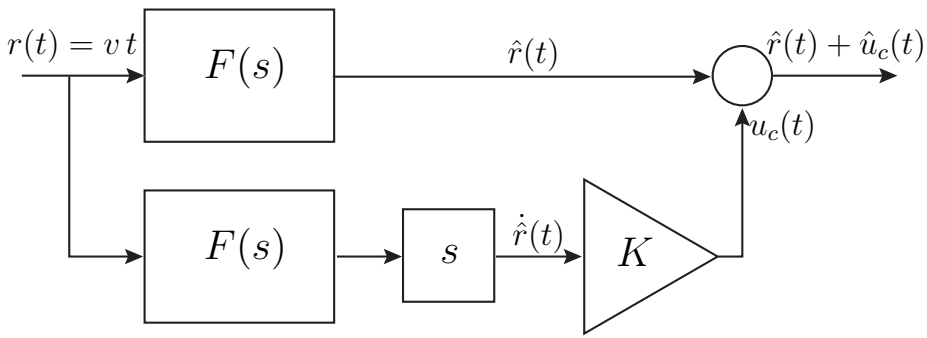

Figure 4: Scheme for asymptotic perfect tracking of a ramp reference input equivalent to the scheme of Fig. 3.

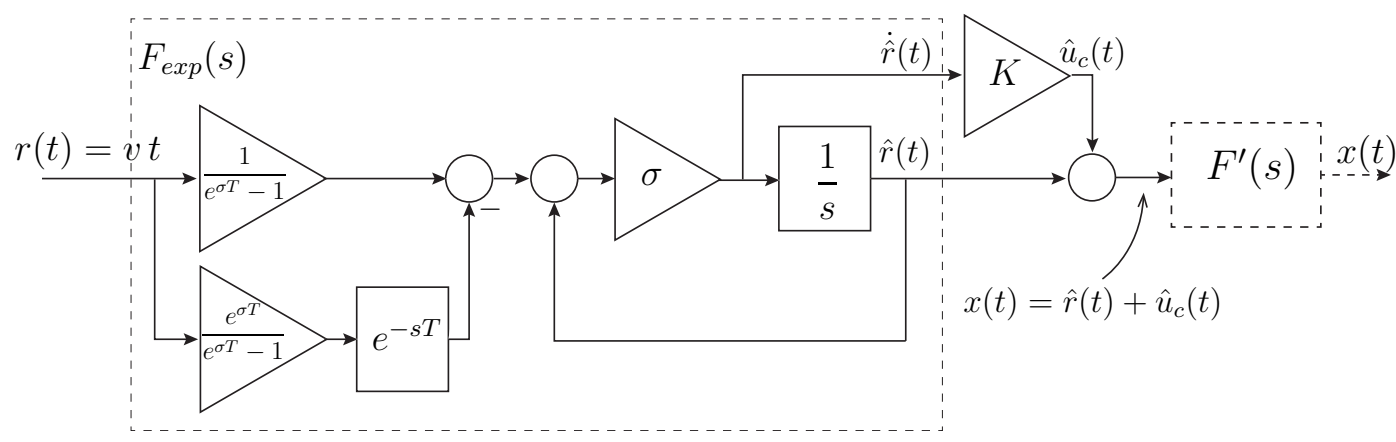

Figure 5: Scheme for asymptotic perfect tracking of a ramp reference input with an exponential smoother.

signal but also provides its first derivative, its use solves the problem of the causality of the compensation scheme reported in Fig. 3. In fact, because of the linearity, the general scheme of Fig. 3 can be transformed as in Fig. 4, and by considering the expression of the exponential smoother the structure reported in Fig. 5 is finally obtained. Note that the scheme can be seen as the composition of a two-impulse input shaper, responsible for vibration suppression, a first-order low-pass filter, that increases the continuity of the input signal and allows the computation of its first derivative, and finally 




Figure 6: Profiles of $\alpha(\delta, k)$ defined in (8) as a function of $\delta$ for different values of $k$. the tracking error compensator based on the gain $K$, which includes the gain $K_{\text {exp }}$ of the exponential smoother and the contributions of the other dynamical systems of the chain such as the additional filters, denoted by $F^{\prime}(s)$, and the plant $G(s)$. The computation of $K_{\text {exp }}$ can be performed according to the definition in (3), i.e.

$$
K_{\text {exp }}=-\lim _{s \rightarrow 0} \frac{d F_{\text {exp }}(s)}{d s}=\frac{e^{\sigma T}(1-\sigma T)-1}{\sigma\left(1-e^{\sigma T}\right)} .
$$

By assuming that the choice of the filter parameters is made according to (6) and (7), it is possible to see that $K_{\text {exp }}$ is a linear function of $T_{0}$ according to a multiplicative parameter that only depends on $\delta$ and $k$, i.e.

$$
K_{\text {exp }}=\alpha(\delta, k) k T_{0}, \quad \text { with } \alpha(\delta, k)=\frac{e^{\zeta}(1-\zeta)-1}{\zeta\left(1-e^{\zeta}\right)}
$$

where

$$
\zeta=-k \frac{2 \pi \delta}{\sqrt{1-\delta^{2}}}
$$

In Fig. 6 the parameter $\alpha$ as a function of $\delta$ and $k$ is shown. Note that the for small values of $\delta$ the coefficient $\alpha(\delta, k)$ is marginally influenced by $k$, and 
when $\delta=0, \alpha(0, k)=\frac{1}{2} \forall k$.

In Fig. 7 the response of the system (4) with $\omega_{n}=15 \mathrm{rad} / \mathrm{s}$ and $\delta=0.1$ to a ramp signal is shown. The straightforward application of $r(t)$ leads to large oscillations, see Fig. 7(a) and in particular the profile of $\varepsilon_{y}(t)$ representing the tracking error, i.e. $\varepsilon_{y}(t)=r(t)-y(t)$. When $r(t)$ is filtered according to the scheme of Fig. 5, where $F^{\prime}(s)=1$ and $K=K_{\text {exp }}$, the plant output is no longer affected by residual vibrations but, while after the initial transient the tracking error $\varepsilon_{x}(t)=r(t)-x(t)$ between the filtered ramp $x(t)$ and the original signal $r(t)$ goes to zero, the error $\varepsilon_{y}(t)$ does not vanish and tends to a constant value, see Fig. 7(b). This is due to the fact that the compensation gain $K$ does not take into account the contribution of the plant $\left(K_{\text {plant }}=2 \delta / \omega_{n}\right)$. If the gain $K=K_{\text {exp }}+K_{\text {plant }}$ is considered, the filtered signal $x(t)$ allows the plant to exactly track the reference input $r(t)\left(\varepsilon_{y} \rightarrow 0\right)$ even if, after the transient, the filtered ramp differs from $r(t)$, see Fig. 7(c). It is worth noting that the use of the exponential smoother makes the duration of the transient terms equal to $T$, computed according to (7), which in this application is $0.421 \mathrm{~s}$. This is due to the fact that $T$ is exactly the duration of the impulse response of $F_{\exp }(s)$, whose expression is

$$
f_{\text {exp }}(t)=\frac{\sigma}{e^{\sigma T}-1} e^{\sigma t} m(t), \quad \text { with } m(t)= \begin{cases}1, & 0 \leq t \leq T \\ 0, & \text { otherwise }\end{cases}
$$

In Fig. 8, the profiles of $f_{\text {exp }}(t)$ obtained for different values of $\sigma$ are shown. When $\sigma=0$ the impulse response of the exponential filter becomes a rectangular function of amplitude $1 / T$. As a matter of fact, exponential smoothers represent a generalization of so-called rectangular smoothers, whose transfer 


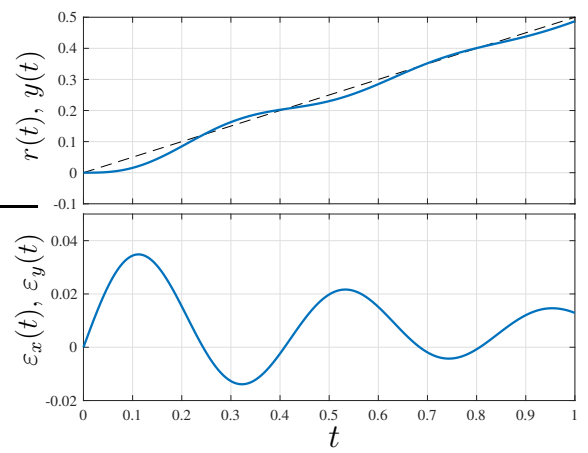

(a)

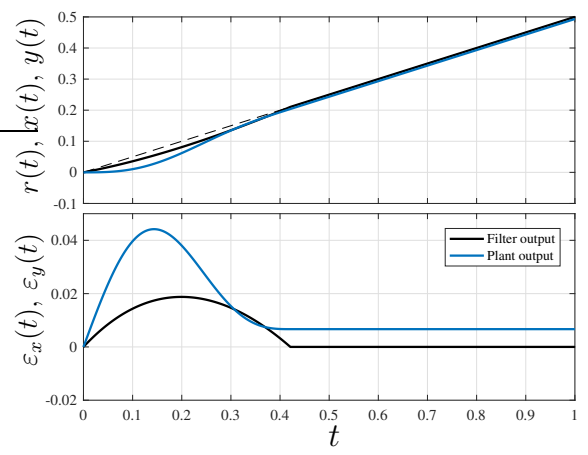

(b)

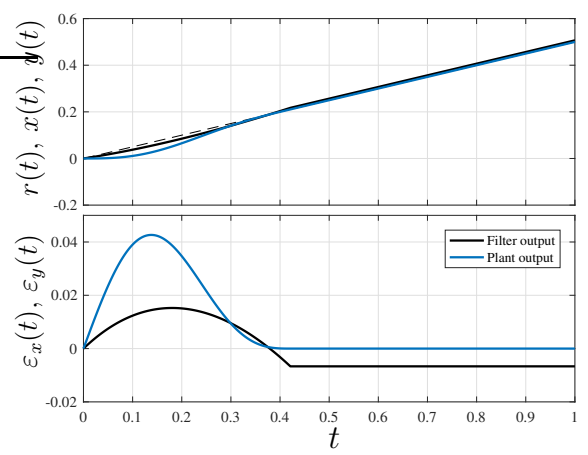

(c)

Figure 7: Response of the vibratory plant (4) to different inputs and related tracking errors $\varepsilon(t)$ : simple input ramp $r(t)$ (a), smoothed input ramp with the compensator gain $K=K_{\text {exp }}$ (b) and smoothed input ramp with the compensator gain $K=K_{\text {exp }}+K_{\text {plant }}$ (c). 


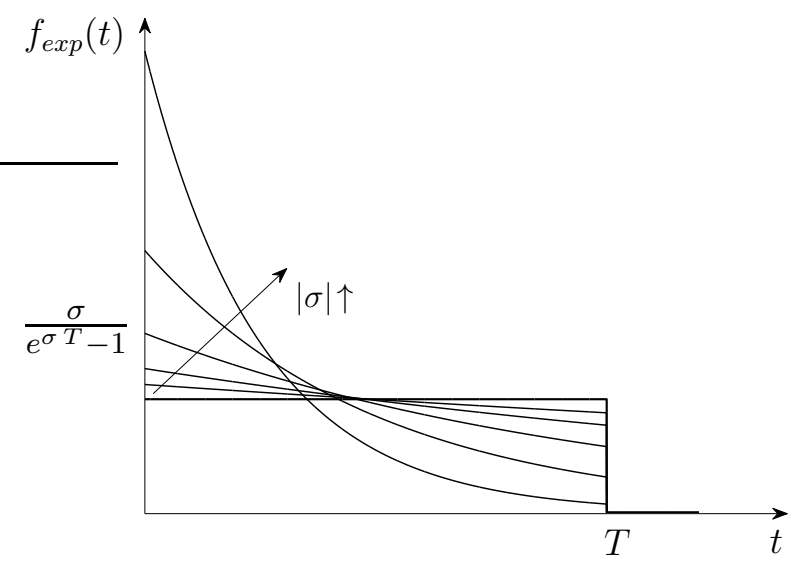

Figure 8: Impulse response of $F_{\text {exp }}(s)$ for negative values of parameter $\sigma$.

function is

$$
F_{r e c}(s)=\frac{1-e^{-s T}}{T s}
$$

which have been used in Biagiotti and Melchiorri (2012) to plan multisegment polynomial trajectories compliant with given bounds on their derivatives.

\section{Compliance with kinematic constraints}

The basic scheme for perfect asymptotic tracking of ramp input signals outlined in the previous section guarantees the suppression of the residual vibration but not the compliance with constraints on velocity, acceleration, etc. To this end, it is possible to act on the parameters of the exponential filter. Since they are determined according to (6) and (7) on the basis of the plant characteristics, the unique free parameter of the filter remains the integer number $k$ in (7) which ranges from 1 to $\infty$. Unfortunately, the value of $k$ determines the duration of the impulse response of the filter, and therefore of the transient, and for this reason, although different choices are possible, 
it is usually assumed equal to one. In order to understand the effect of $k$ on the filtered signal derivatives, some preliminary considerations are necessary. As already mentioned, the exponential filter acts on the input signal by increasing its smoothness by one. For instance, when a unit step signal, which is a discontinuous function with unbounded first derivative, is applied to $F_{\text {exp }}(s)$, the velocity related to the output signal is exactly $f_{\exp }(t)$ and therefore it is bounded in magnitude. From Fig. 8, it is possible to notice that, for a given value of $T$, the maximum value of the velocity produced by the exponential smoother (for $t=0$ ) grows with $|\sigma|$, while its minimum nonzero value, i.e. for $t=T$, decreases.

If the parameters of the filter are selected according to (6) and (7), the limit values $\max _{t \in[0, T]} f_{\text {exp }}(t)$ and $\min _{t \in[0, T]} f_{\text {exp }}(t)$ are linear functions of $1 / T$ according to coefficients which only depend on $\delta$ and $k$ :

$$
\begin{aligned}
\max _{t \in[0, T]} f_{\text {exp }}(t) & =\gamma(\delta, k) \frac{1}{T}, & \min _{t \in[0, T]} f_{\text {exp }}(t) & =\varphi(\delta, k) \frac{1}{T} \\
\gamma(\delta, k) & =\frac{\zeta}{e^{\zeta}-1}, & \varphi(\delta, k) & =\frac{\zeta e^{\zeta}}{e^{\zeta}-1}
\end{aligned}
$$

where $\zeta$ is defined in (9). In Fig. 9, the functions $\gamma(\delta, 1)$ and $\varphi(\delta, 1)$ are shown as a function of $\delta$ (while it is assumed $k=1$ ). For small values of $\delta$, the value of $k$ influences marginally the two functions. In particular, $\gamma(0, k)=1$ and $\varphi(0, k)=1, \forall k$.

If the reference signal is of class $\mathcal{C}^{-1}$, i.e. discontinuous like the signal composed by step and ramp functions, the exponential smoother can only bound its velocity. Accordingly, in order to impose desired limit values on higher order derivatives it is necessary to introduce supplemental filters in the chain that, in the scheme of Fig. 5, are included in the function $F^{\prime}(s)$. 


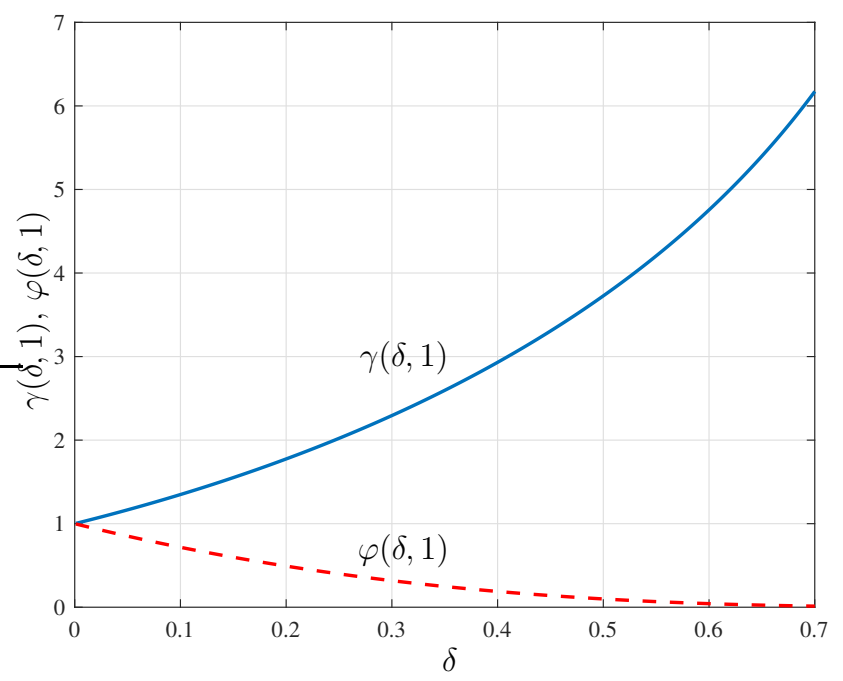

Figure 9: Profiles of functions $\gamma(\delta, k)$ and $\varphi(\delta, k)$ defined in (12) as a function of $\delta(k=1)$.

In particular, the rectangular smoothers, whose transfer function is reported in (10), are used. With a proper choice of the parameter $T_{i}$, each smoother can guarantee the compliance with an additional kinematic bound. As a consequence,

$$
F^{\prime}(s)=F_{r e c, 1}(s) \cdots F_{r e c, n}(s)
$$

where $n$ is the number of constraints that have to be met besides velocity. In order to define the best filter structure and the best set of parameters values in case of constraints on the first $n+1$ derivatives of the signal $x(t)$, it is convenient to consider two different scenarios, which are very common in practical applications:

1. the reference signal is composed by a sequence of ramp functions, see Fig. 10(a);

2. the reference signal has a sawtooth profile, defined by the combination of ramp and step functions, see Fig. 10(b). 


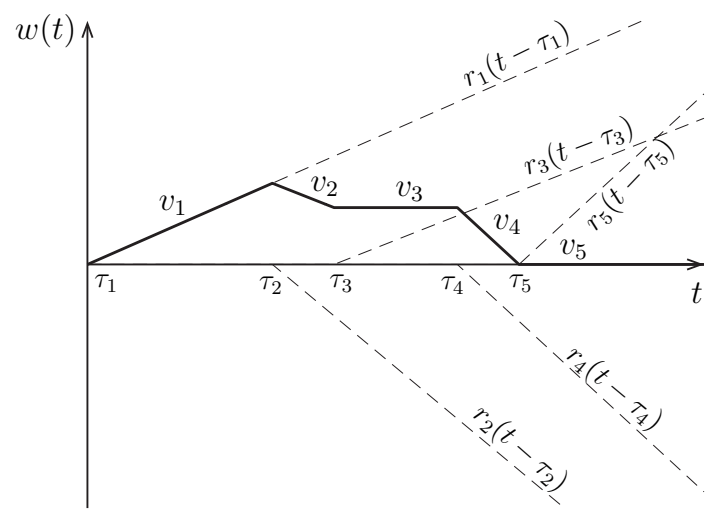

(a)

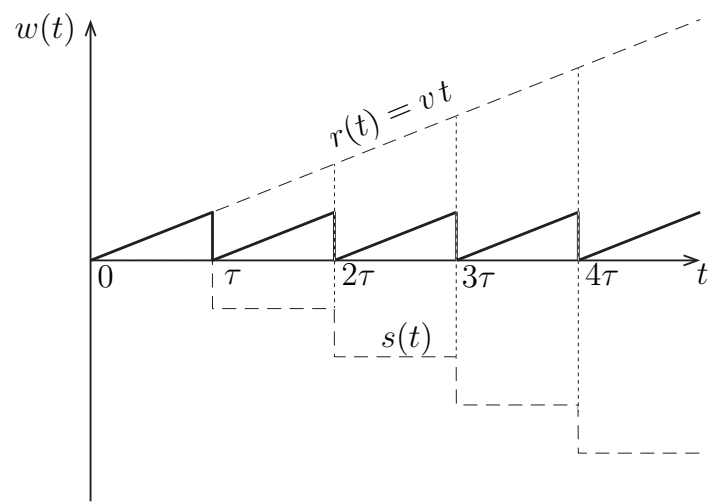

(b)

Figure 10: Generic input profile $w(t)$ obtained by a combination of ramp functions (a) and decomposition of a sawtooth profile in its elementary components (b).

First the problem of the velocity limitation, which is related to the choice of the parameters of the exponential filter, is considered and then the further constraints, requiring additional smoothers, are taken into account.

\subsection{Sequence of ramp functions}

The piecewise linear function of Fig. 10(a) can be decomposed into a sequence of ramp functions as 


$$
\begin{aligned}
w(t)= & r_{1}\left(t-\tau_{1}\right)+r_{2}\left(t-\tau_{2}\right)+r_{3}\left(t-\tau_{3}\right)+\ldots \\
= & \left(v_{1}-v_{0}\right)\left(t-\tau_{1}\right)+\left(v_{2}-v_{1}\right)\left(t-\tau_{2}\right)+ \\
& +\left(v_{3}-v_{2}\right)\left(t-\tau_{2}\right)+\ldots
\end{aligned}
$$

where $v_{i}$ denotes the velocity of the tract starting at time-instant $\tau_{i}\left(v_{0}=0\right)$. When $w(t)$ is applied to the scheme of Fig. 5, a change in the filtered signal occurs at every time instant $\tau_{i}$, when a new ramp is applied, i.e. at the transition between a segment and the subsequent. If a generic transition between the $(i-1)$-th and the $i$-th tract is considered, like in Fig. 11, the velocity of the filtered signal $x(t)$ is given by the contribution of the ramp and of the compensating term, i.e. $\dot{x}(t)=\dot{\hat{r}}(t)+\dot{\hat{u}}_{c}(t)$. As shown in the figure, its maximum (absolute) value occurs at time instant $t_{\max }=\tau_{i}+T$ and can be computed as

$$
\max \{|\dot{x}(t)|\} \leq\left|v_{i}+K\left(v_{i}-v_{i-1}\right) \varphi(\delta, k) \frac{1}{T}\right|
$$

where $\varphi(\delta, k)$ is defined in $(12)$ and $K=K_{\exp }\left(+K_{\text {plant }}\right)$. The contribution of the plant must be taken into account in order to exactly guarantee $\varepsilon_{y} \rightarrow 0$, but when the system is poorly damped, like in the typical applications requiring vibration suppression techniques, the gain becomes negligible. With this in mind, in the following the gain $K_{\text {plant }}$ will be omitted. As regard equation (14) it is worth noticing that, as shown in (8), $K_{\text {exp }}$ depends on $\delta$, $T_{0}$ (both constant) and on the free parameter $k$. Therefore, in principle, by acting on $k$ it is possible to modify the peak value of the velocity, but it is necessary to recall that the actual duration of the filter impulse response, $T=k T_{0}$, grows with $k$ and, consequently, cannot be augmented arbitrarily. Because of the discontinuities on $\dot{x}(t)$ shown in Fig. 11(c), the corresponding 


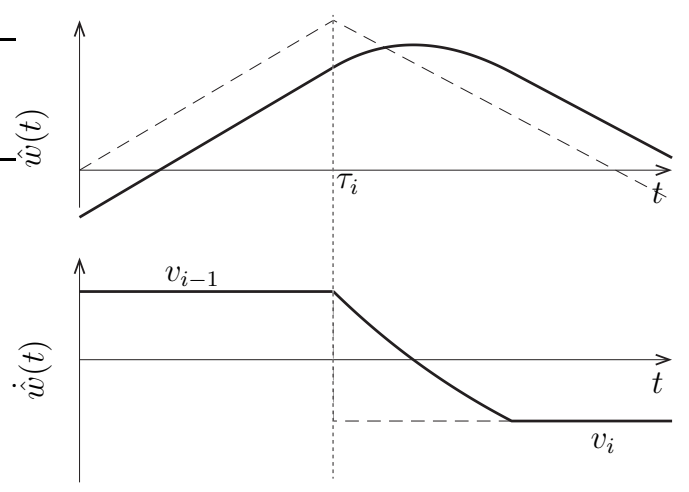

(a)

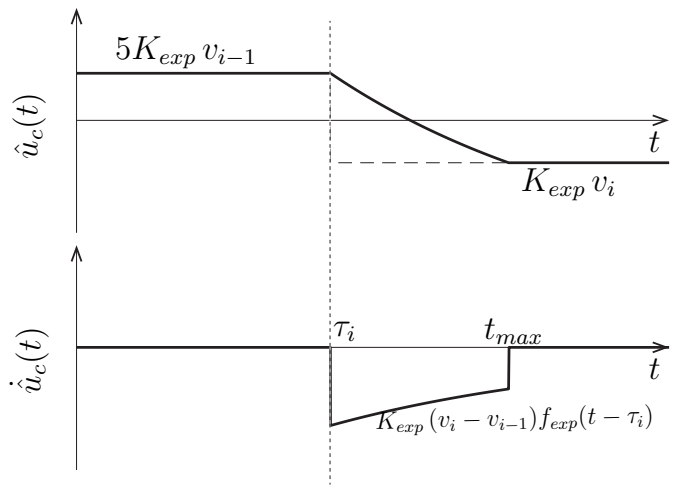

(b)

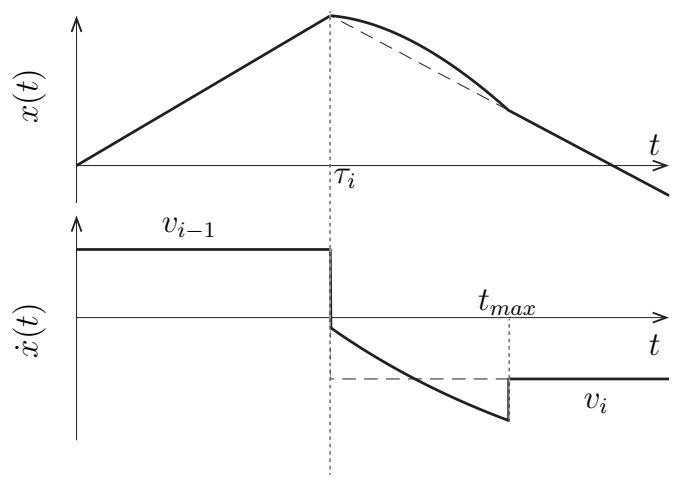

(c)

Figure 11: Position and velocity profiles of a tract of the filtered reference signal $w(t)$ composed by ramps (a), of the compensation control action $\hat{u}_{c}(t)$ (b) and of their combination $x(t)=\hat{w}(t)+\hat{u}_{c}(t)(\mathrm{c})$ when the exponential smoother is applied alone. 


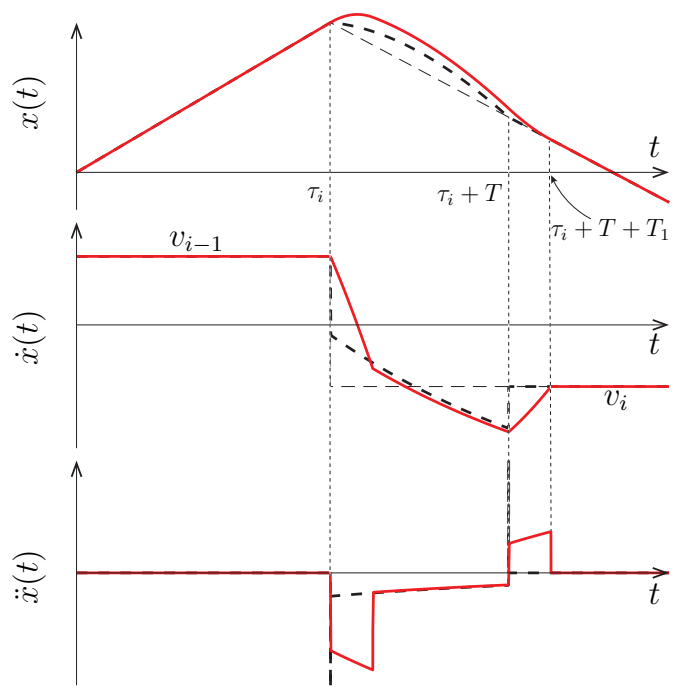

Figure 12: Position, velocity and acceleration profiles of a tract of the signal $w(t)$ composed by ramp functions filtered by the feed-forward controller of Fig. 5 with an additional rectangular filter $F_{r e c, 1}(s)$ (solid red line). The dashed black line denotes the signal $x(t)$ in Fig. 11(c).

acceleration is unbounded. For this reason, in order to limit its peak value a rectangular smoother, whose transfer function is reported in (10), must be included in the cascade. The effects of this filter on $x(t)$ and its derivatives are shown in Fig. 12. Although the rectangular smoother cannot increase the peak value of the derivatives of the filtered signal as proved in Biagiotti and Melchiorri (2012), from Fig. 12 it comes out that the velocity of the new signal $x(t)$ becomes higher. This is not due to the additional filtering action but to the compensation term whose gain must take into account also the contribution of $F_{r e c, 1}(s)$. In fact, inequality (14) remains valid but it is necessary to consider

$$
K=K_{e x p}+K_{r e c, 1}, \quad \text { with } K_{r e c, 1}=\frac{T_{1}}{2}
$$


being $T_{1}$ the duration of the impulse response $F_{r e c, 1}(s)$.

Thanks to the new smoother, also $\ddot{x}(t)$ is bounded and its peak value can be estimated as

$$
\max \{|\ddot{x}(t)|\} \leq\left|\left(v_{i}-v_{i-1}\right) \frac{1}{T} \gamma(\delta, k)\left(1+\frac{K}{T_{1}}\right)\right| .
$$

If the specifications also prescribe a limited jerk, it is necessary to add a second rectangular smoother $F_{r e c, 2}(s)$. In this case, inequalities (14) and (16) remain valid provided that $K$ takes into account also $K_{r e c, 2}$, while the jerk is limited by

$$
\max \left\{\left|x^{(3)}(t)\right|\right\} \leq \frac{\max \left\{\left|x^{(2)}(t)\right|\right\}}{T_{2}}=\left|\left(v_{i}-v_{i-1}\right) \frac{1}{T} \gamma(\delta, k)\left(1+\frac{K}{T_{1}}\right) \frac{1}{T_{2}}\right| .
$$

The procedure can be further iterated by considering additional rectangular smoothers, which imply the inequalities

$$
\max \left\{\left|x^{(i+1)}(t)\right|\right\} \leq \frac{\max \left\{\left|x^{(i)}(t)\right|\right\}}{T_{i}}, \quad i=3, \ldots, n .
$$

Finally, given the kinematic constraints, the number of required smoothers is firstly selected and then the parameters that guarantee the compliance with these bounds are computed on the basis of relations (14)-(18).

If for instance the constraints on velocity and acceleration

$$
\max \{|\dot{x}(t)|\} \leq \mathrm{v}_{\max } \text { and } \max \{|\ddot{x}(t)|\} \leq \mathrm{a}_{\max }
$$

are considered, like in the experimental tests illustrated in Sec. 5, a second order filter composed by an exponential and a rectangular smoother should be considered. In this case, even if the inequality (14) is valid, the bound on 
the velocity can be refined as

$$
\max \{|\dot{x}(t)|\} \leq\left|v_{i}+\left(v_{i}-v_{i-1}\right) \frac{1}{T}\left(\gamma(\delta, k) K-\varphi(\delta, k) \frac{T_{1}}{2}\right)\right|
$$

Note that (20) is not true for higher order filters.

From (20) and (16) the nonlinear algebraic system

$$
\begin{aligned}
\frac{1}{T}\left(\gamma(\delta, k) K-\varphi(\delta, k) \frac{T_{1}}{2}\right) & \leq \frac{\mathrm{v}_{\max }-\left|v_{i}\right|}{\left|v_{i}-v_{i-1}\right|} \\
\frac{1}{T} \gamma(\delta, k)\left(1+\frac{K}{T_{1}}\right) & \leq \frac{\mathrm{a}_{\max }}{\left|v_{i}-v_{i-1}\right|}
\end{aligned}
$$

is obtained, whose solution, if any, provides the values of $k$ and $T_{1}$ which make the signal $x(t)$ compliant with the constraints.

The case in which $\delta \approx 0$, due to the fact that the system is poorly damped or it is deliberately chosen to neglect the damping coefficient in the filter design since its sensitivity to variation on $\delta$ is rather limited (see Biagiotti et al. (2016)), is particularly attractive being the final motion a standard multi-segment polynomial trajectory. Moreover, as already mentioned, if $\delta \approx$ 0 the compensation term due to the plant becomes negligible, i.e. $K_{\text {plant }} \approx 0$. With this assumption $\varphi(\delta, k)=\gamma(\delta, k)=1$ and $K_{\text {exp }}=\frac{T}{2}$. As consequence, equations (20) and (16) become

$$
\begin{aligned}
& \max \{|\dot{x}(t)|\}=\left|v_{i}+\frac{1}{2}\left(v_{i}-v_{i-1}\right)\right| \\
& \max \{|\ddot{x}(t)|\}=\left|\left(v_{i}-v_{i-1}\right)\left(\frac{3}{2 T}+\frac{1}{2 T_{1}}\right)\right| .
\end{aligned}
$$

Note that, in this case, the estimation of the peak values is exact. From (21) it descends that the maximum value of the velocity $\dot{x}(t)$ cannot be influenced 

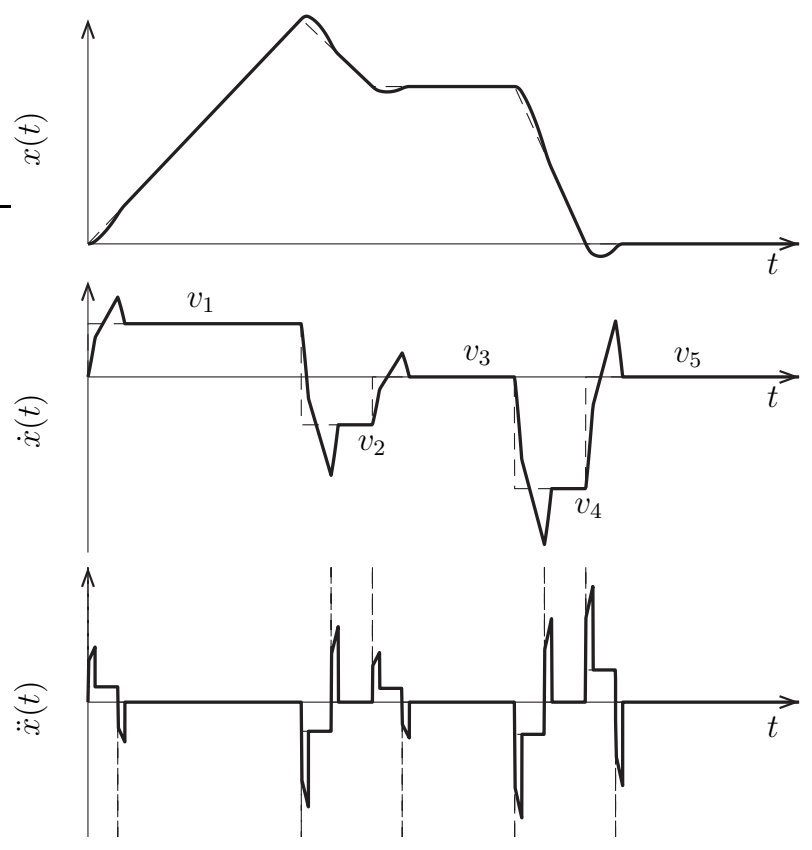

Figure 13: Position, velocity and acceleration profiles of the signal $x(t)$ obtained by filtering the reference signal $w(t)$ composed by ramp functions shown in Fig. 10(a) with the compensator obtained for $\delta=0$.

by the feed-forward controller. Therefore, it is necessary to select the actuator on the basis of the values found with (21) by considering the velocities $v_{i}$ of the reference signal. Conversely, for a given actuator characterized by the maximum velocity $\mathrm{v}_{\max }$ it is necessary to modify the values of $v_{i}$ if (21) is not met. The acceleration can be limited by acting on both the smoothers, since from (22) it descends that

$$
\frac{3}{2 T}+\frac{1}{2 T_{1}} \leq \frac{\mathrm{a}_{\max }}{\left|v_{i}-v_{i-1}\right|} .
$$

Therefore, by increasing $T$ (by acting on $k$ ) and $T_{1}$ the inequality can be easily verified. Figure 13 shows the filtered signal obtained with $\delta=0$ by 


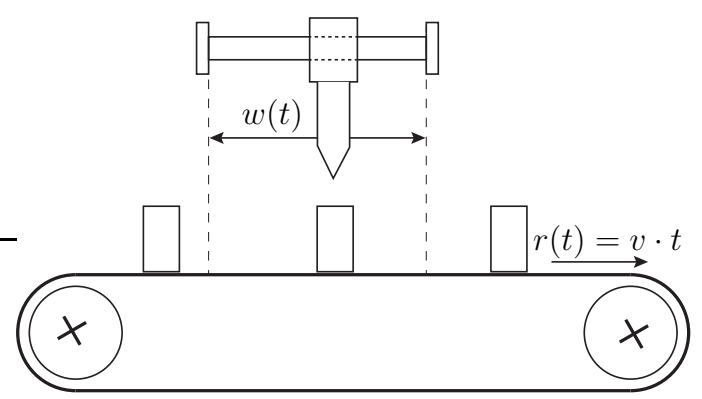

Figure 14: Sketch of a tool operating on items moving on a conveyor belt.

considering the whole reference signal $w(t)$ in Fig. 10(a).

\subsection{Sawtooth reference input}

As shown in Fig. 10(b), a reference signal with a sawtooth profile can be decomposed into the sum of a ramp function and a staircase function, i.e.

$$
\begin{aligned}
w(t) & =r(t)+\sum_{i=1}^{\infty} A_{i} h(t-i \tau) \\
& =v \cdot t-\sum_{i=1}^{\infty} v \tau h(t-i \tau)
\end{aligned}
$$

where $h(t)$ denotes the unit step signal, $v$ is the slope of the ramp function and $\tau$ is the period of the sawtooth signal. Note that the reference trajectory described by (24) is very significant for industrial applications since it represents the typical profile of an intermittent movement synchronized with an axis moving at a constant velocity. This is what happens for instance when a tool must operate on items moving at a constant velocity on a conveyor belt, as roughly sketched in Fig. 14 .

A second order filter, obtained by combining the exponential smoother with a rectangular filter, is initially considered. Apart the initial transient, the velocity of the filtered signal $x(t)$, that in steady state becomes $v$, only 


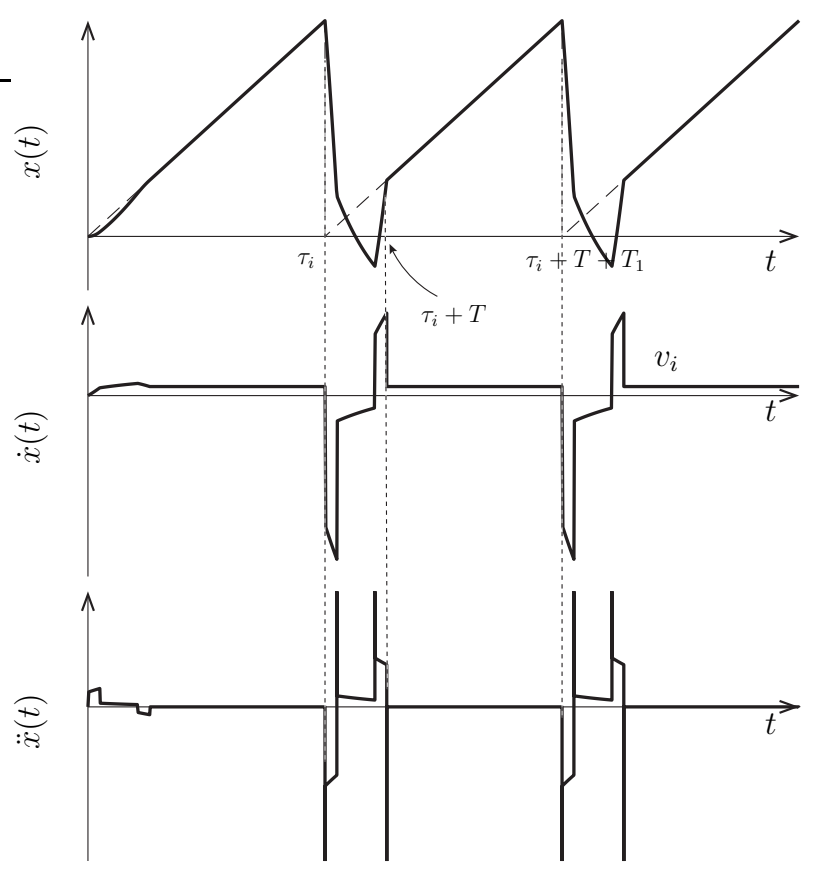

Figure 15: Position, velocity and acceleration profiles of the sawtooth reference signal (24) filtered with the scheme of Fig. 5.

changes at time instants $i \tau$ because of the application of the step functions $h(t-i \tau)$. Being the step a discontinuous function and the filter composed by two smoothers, on the basis of simple considerations the continuity of $\dot{x}(t)$ would be expected, but as shown in Fig. 15 the velocity of the filtered signal is affected by some discontinuities. Moreover, with respect to the initial sawtooth reference signal the position trajectory $x(t)$ exhibits a quite evident undershoot. Both effects are due to the fact that the compensation mechanism of the phase delay, outlined in Sec. 2, acts not only on the ramp function composing the input but also on the train of step functions. For this reason, a compensation scheme which takes into account only the contribution of the ramp must be adopted. Since the signal $w(t)$ includes a single 


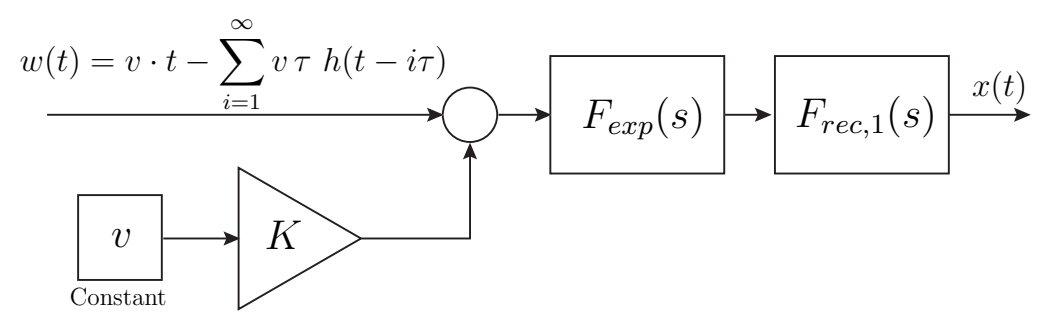

Figure 16: Filter for asymptotic perfect tracking of a sawtooth reference input with velocity and acceleration constraints $\left(K=K_{e x p}+K_{r e c, 1}\right)$.

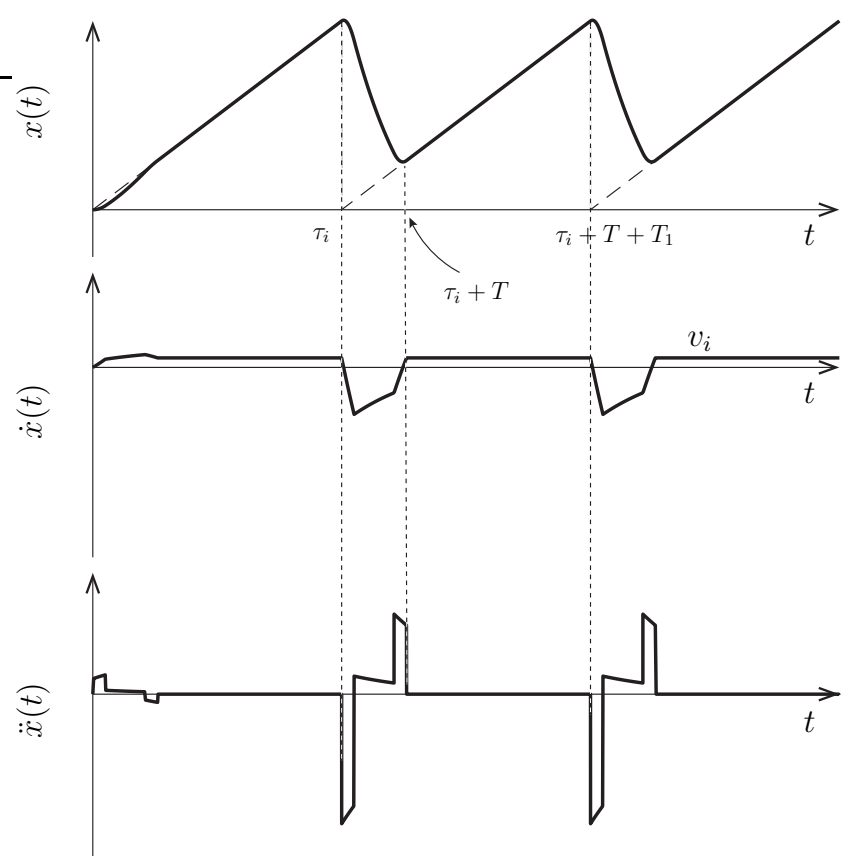

Figure 17: Position, velocity and acceleration profiles of the sawtooth reference signal (24) filtered with the scheme of Fig. 16.

ramp with constant velocity $v$, the compensator takes the form reported in Fig. 16, where a simple additional term proportional to $v$ is added to the reference input and both are filtered by $F_{\text {exp }}(s) F_{r e c, 1}(s)$. With this compensation scheme, the filtered signal $x(t)$ becomes a function of class $\mathcal{C}^{1}$, as shown in Fig. 17, and the peak values of velocity and acceleration meet the 
following inequalities

$$
\begin{aligned}
& \max \{|\dot{x}(t)|\} \leq\left|v\left(1-\gamma(\delta, k) \frac{\tau}{T}\right)\right| \\
& \max \{|\ddot{x}(t)|\} \leq\left|v \gamma(\delta, k) \frac{\tau}{T T_{1}}\right|
\end{aligned}
$$

Note that the peak values does not depends on the gain $K$ of the compensator. Moreover, as in the case of a sequence of ramp functions, also with the sawtooth reference signal it is possible to bound further derivatives of the filtered signal $x(t)$, by integrating additional smoothers in $F^{\prime}(s)$. If $n$ rectangular smoothers are considered, the signal derivatives are limited up to the $(n+1)$-th order, and the bounds can be expressed as

$$
\max \left\{\left|x^{(i)}(t)\right|\right\} \leq\left|v \gamma(\delta, k) \frac{\tau}{T \sum_{j=1}^{i-1} T_{j}}\right|, \quad i=2, \ldots, n+1 .
$$

Also with the sawtooth reference signal the case $\delta \approx 0$ is particularly important, since in this case the vibration suppression capabilities of the smoothers are combined with the simplicity of standard multi-segment trajectories like trapezoidal velocity profiles.

With this hypothesis, the peak values can be exactly predicted, i.e.

$$
\begin{aligned}
& \max \{|\dot{x}(t)|\}=\left|v\left(1-\frac{\tau}{T}\right)\right| \\
& \max \{|\ddot{x}(t)|\}=\left|v \frac{\tau}{T T_{1}}\right| .
\end{aligned}
$$

Given the kinematic constraints (19), the parameters of the two smoothers composing the filter of Fig. 16 must comply the constraints

$$
T \geq \frac{|v| \tau}{|v|+\mathrm{v}_{\max }}, \quad T_{1} \geq \frac{|v|+\mathrm{v}_{\max }}{\mathrm{a}_{\max }}
$$


and accordingly the optimal values of the parameters, that minimize the duration of the transient, are

$$
k=\operatorname{ceil}\left(\frac{|v|}{|v|+\mathrm{v}_{\max }} \frac{\tau}{T_{0}}\right), \quad T_{1}=\frac{|v|+\mathrm{v}_{\max }^{\star}}{\mathrm{a}_{\max }}
$$

where $\mathrm{v}_{\max }^{\star}$ denotes the maximum velocity actually reached by the reference signal with the selected value of $k$, i.e. $\mathrm{v}_{\text {max }}^{\star}=\max _{k=k^{\star}}\{|\dot{x}(t)|\}$.

\section{Experimental tests}

In order to validate the proposed method the experimental setup of Fig. 18 has been used. It is based on a thin stainless steel flexible link directly connected to the slider of a linear motor. The side of the link connected to the motor is instrumented with a strain gauge that can detect the local deformation of the beam. The linear motor LinMot PS01-37x120 with the servo controller LinMot E2010-VF have been used (see LinMot (2019)). The servo controller performs the basic current control, while a standard PC with a Pentium IV $3 \mathrm{GHz}$ processor equipped with a Sensoray 626 data acquisition board is in charge of the position control based on a PID controller and a feedforward action. The real-time operating system RTAI-Linux on a Debian SID distribution with Linux kernel 2.6.17.11 and RTAI 3.4 allows the position controller to run with a sampling period $T_{s}=500 \mu \mathrm{s}$. For the design of the control scheme and of trajectory generator, the MatLab/Simulink/RealTime Workshop environment has been used. The generation of the reference signal composed by ramp functions and the proposed filtering action based on exponential and rectangular smoothers have been implemented in the same (Simulink) control scheme that performs the motor position control. For this 


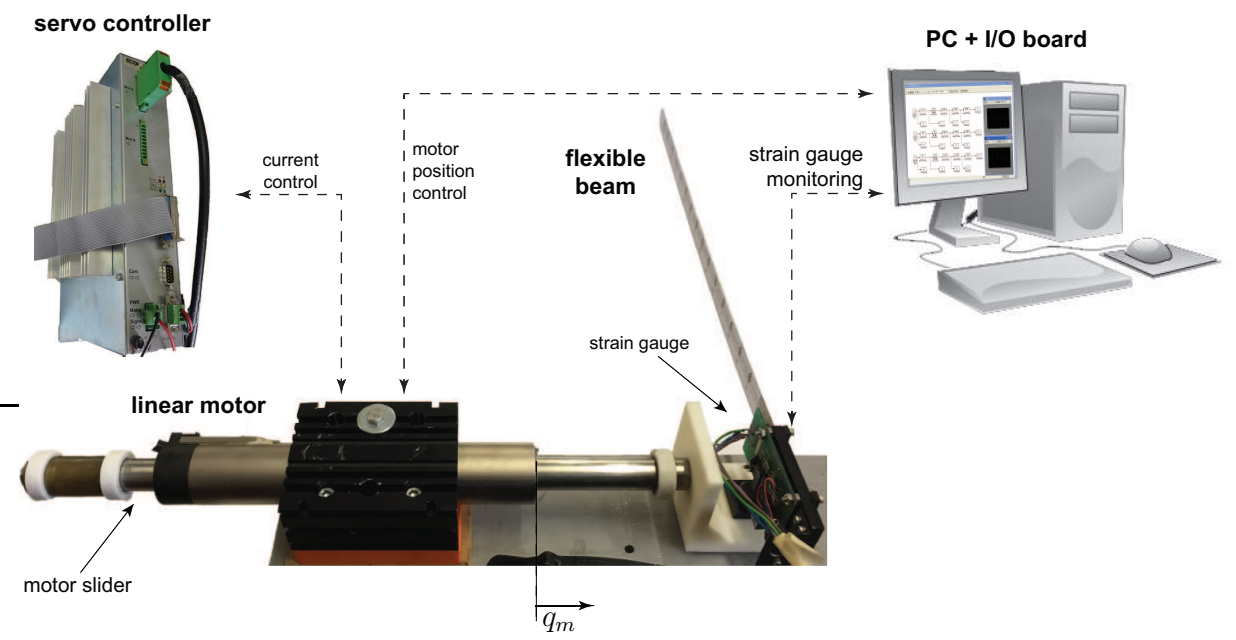

Figure 18: Experimental setup based on a flexible link.

reason the discrete-time expression of the proposed filter, with sampling-time $T_{s}$, must be used. This can be obtained by substituting in the schemes shown in Fig. 5 and in Fig. 16 the discrete-time expressions of the smoothers which are reported in Appendix A. Note that, even in this case the proposed feedforward control is implemented in the same scheme performing the position control of the plant, in a modern architecture for the control of e.g. automatic machines, which may take advantage from the proposed technique, the filter can be implemented as a stand-alone module, written starting from the difference equations corresponding to the discrete-time transfer functions of the smoothers, that receives the signal composed by ramp functions as an input and provides online the filtered output, which can be sent to the servo drives controlling the position of the actuators.

The plant can be modeled as in Fig. 19. The dynamics of the link subject to the lateral acceleration $\ddot{x}$ of the motor is described by a partial differential equation Luo et al. (1995). The assumption of separability of spatial and 


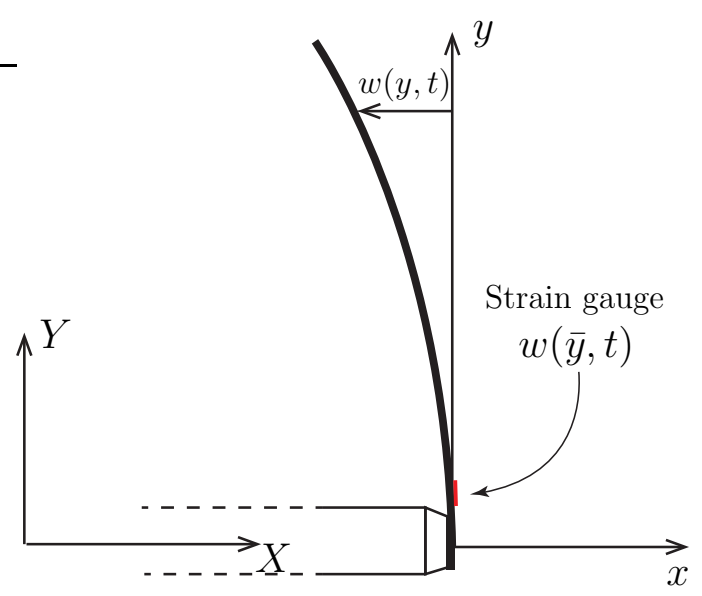

Figure 19: Model of the uniform cantilever beam used in the experiments.

temporal variables allows to obtain a closed form solution of the bending deformation

$$
w(y, t)=\sum_{i=1}^{\infty} \psi_{i}(y) q_{i}(t)
$$

where $\psi_{i}(y)$ are the mode shapes depending on the boundary conditions imposed by the physical system, and $q_{i}(t)$ are the generalized modal coordinates, oscillatory in time according to the frequency $\omega_{i}$ Kane et al. (1987); Bellezza et al. (1990). Even if the expression (31) takes into account infinite terms, in practice only few modes are meaningful for the bending of the beam. In particular, the flexible link used in the experiments is strongly affected by two modes located at $\omega_{r 1} \approx 20.18 \mathrm{rad} / \mathrm{s}$ and $\omega_{r 2} \approx 127.5 \mathrm{rad} / \mathrm{s}$, respectively, while additional contributions are negligible. The dynamic modes are both slightly damped. For instance, the fist mode is characterized by a damping coefficient $\delta=0.0043$. For this reason, the design of the reference trajectory filters is based on the assumption of completely undamped plant and accordingly the exponential smoother has become a simple rectangular smoother. The 


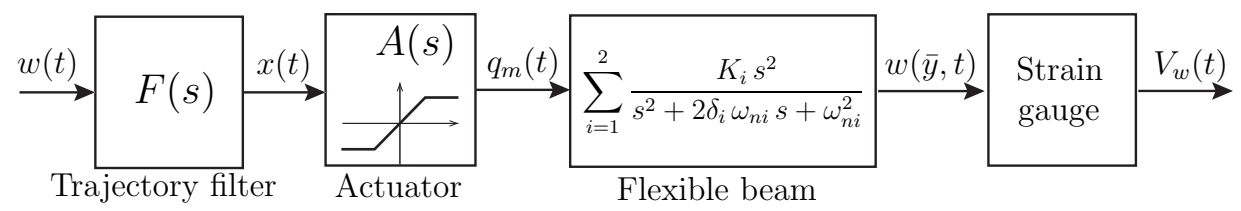

Figure 20: Block-scheme representation of the experimental setup, based on a flexible link, shown in Fig. 18.

strain gauge, sensing the deflection in a specific point of the beam, provides a signal $V_{w}(t)$ proportional to $w(\bar{y}, t)$ that is therefore a linear combination of temporal modes according to the constant coefficients $K_{i}=\psi_{i}(\bar{y})$. In order to asses the level of vibrations, the output voltage $V_{w}(t)$ of the strain gauge is directly used, see Fig. 20.

The rectangular smoother for vibration suppression is designed on the basis of the first resonant mode, i.e. $T_{0}=\frac{2 \pi}{\omega_{r 1}}=0.3114 \mathrm{~s}$. Note that, since $\delta \approx 0$, natural and resonant frequencies of the system are coincident, i.e. $\omega_{r i}=\omega_{n_{i}}$. In order to show the effectiveness of the proposed approach based on smoothers, a comparative evaluation of the performance obtained with some alternative techniques mentioned in the introduction is performed. In particular, on the one hand the so-called signal derivatives limiters, that do not explicitly address the problem of vibration suppression but limit the velocity and acceleration of the input signal, are considered; on the other hand, a $\mathrm{ZVD}^{1}$ input shaper has been designed to suppress the residual vibration, without taking into account kinematic limits. The constraints considered in the experiments are $\mathrm{v}_{\max }=0.1 \mathrm{~m} / \mathrm{s}$ and $\mathrm{a}_{\max }=1 \mathrm{~m} / \mathrm{s}^{2}$.

\footnotetext{
${ }^{1}$ The choice of the ZVD is motivated by the fact that this input shaper produces a delay between input and output equal to $T_{0}$.
} 


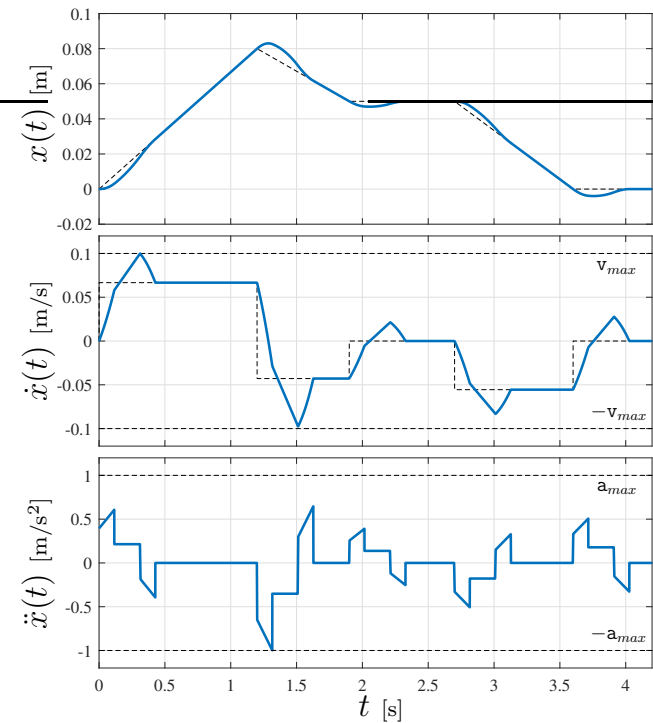

(a)

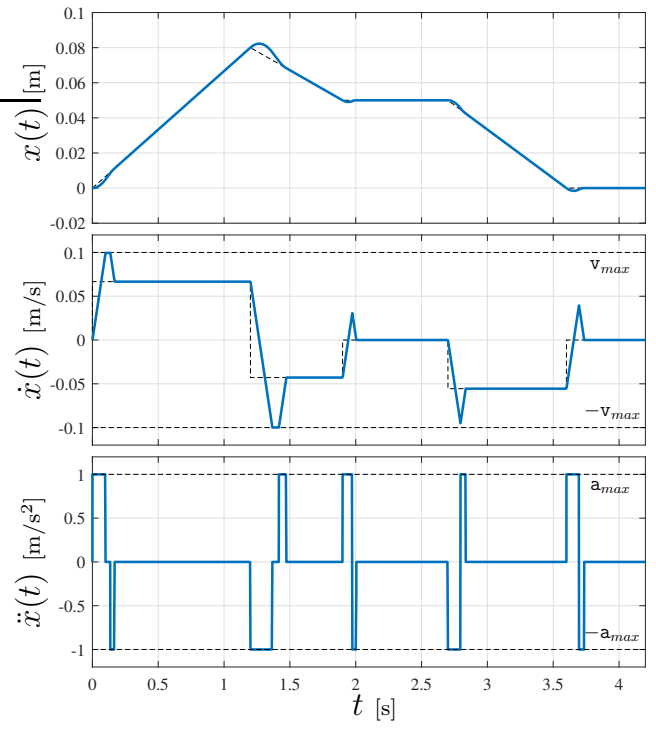

(b)

Figure 21: Position, velocity and acceleration profiles of the sequence of ramp functions, filtered by a cascade of two rectangular smoothers (a) and by a second-order derivatives limiter (b), used in the experiments.

\subsection{Sequence of ramp functions}

The reference signal (13) is defined by

$$
\begin{aligned}
& {\left[v_{i}\right]=[0.0667,-0.0429,0,-0.0556,0] \mathrm{m} / \mathrm{s}} \\
& {\left[\tau_{i}\right]=\left[\begin{array}{lllll}
0, & 1.2, & 1.9, & 2.7, & 3.6
\end{array}\right] \mathrm{s} .}
\end{aligned}
$$

The peak value of the velocity obtained with the cascade of two rectangular filters, and computed with (21), is exactly $0.1 \mathrm{~m} / \mathrm{s}$. The constraints on the acceleration has been met by assuming $T=T_{0}$, i.e. $k=1$, and $T_{1}=0.1159$ $\mathrm{s}$, in accordance with (23). In Fig. 21(a) the profiles of position velocity and acceleration obtained with the proposed filter are shown, and are compared with those of a second-order derivatives limiter, see Fig. 21(b). Note that with the two rectangular smoothers the limit values of velocity and acceler- 


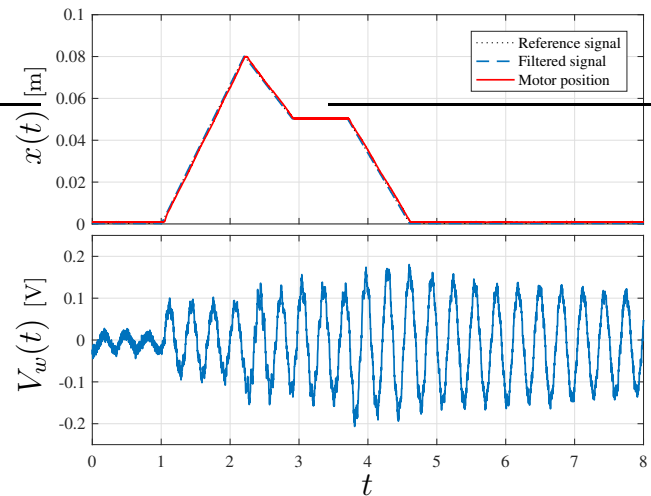

(a)

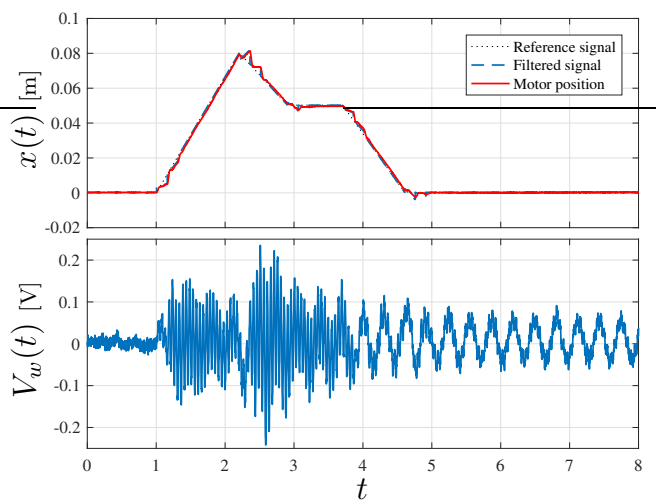

(c)

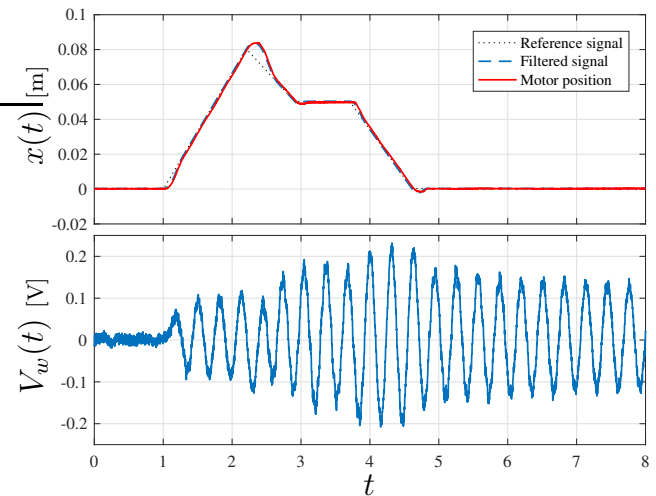

(b)

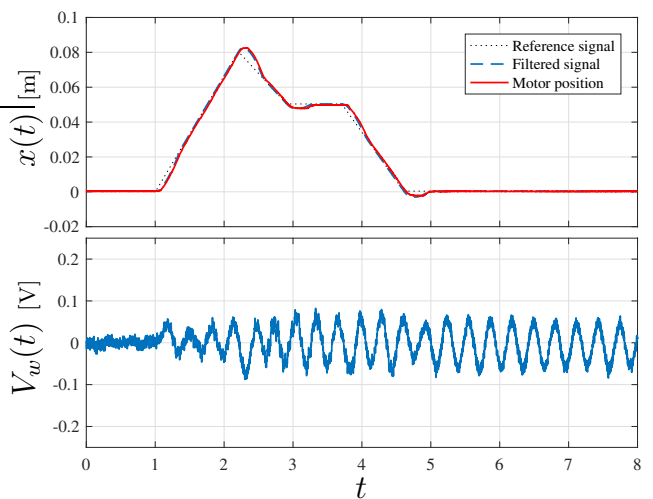

(d)

Figure 22: Reference trajectories obtained by filtering a signal composed by ramp functions with different methods and vibrations induced on the flexible beam: no filters (a), derivatives limiter (b), ZVD Input shaper (c), rectangular smoothers (d).

ation are reached only during the first and second transition between ramp segments, respectively, and that the duration of the different transitions is always constant and equal to $T+T_{1}=0.4273 \mathrm{~s}$. Conversely, the limiter always imposes the minimum-time duration of the transitions which is compliant with the given constraints. In Fig. 22 the results of the different techniques in terms of residual vibration reduction are highlighted. The Figure 22(a) shows the behavior of the flexible beam when no filters are applied to the original ramp sequence. As shown in Fig. 22(b), the use of the derivatives lim- 
iter does not produce significant improvements being the level of the residual vibration essentially unchanged with respect to the direct application of the ramp sequence. Conversely, ZVD input shaper and rectangular smoothers induce a similar reduction of the residual vibration at the end of motion, see Fig. 22(c)-(d). However, during the motion, the amplitude of the signal $V_{w}(t)$ with the ZVD input shaper is considerably higher than the amplitude reached with the smoothers.

These "qualitative" observations are supported by the FFT (Fast Fourier Transform) analysis performed on the signal $V_{w}(t)$ after the end of the trajectory, whose results are reported in Fig. 23 in the form of magnitude spectrum of $V_{w}(j \omega)$. The magnitude of the component at $\omega_{r 1}$ is practically the same without filters or with the derivatives limiter. The reduction of the residual vibration at $\omega_{r 1}$ obtained with the ZVD input shaper and with the smoothers are comparable, being $-62.03 \%$ and $-57.53 \%$ respectively. However, while the smoothers are also able to reduce the vibrations caused by higher frequency modes, because of the low-pass characteristics of their sensitivity curve (see Biagiotti et al. (2016)), the input shaper excites high order modes even more than the original sequence of ramp functions. This effect is highlighted in Fig. 23(c), where the component at $\omega_{r 2}$ is higher in magnitude than the component at the same frequency shown in Fig. 23(a), and it can be explained by considering that the use of the input shaper requires a compensation term composed by a sequence of step functions that produces discontinuities in the reference position profiles, see Fig. 22(c). Because of these discontinuities, during the motion (and not only at the end) the flexible beam is affected by high frequency oscillations. In addition, the discontinu- 


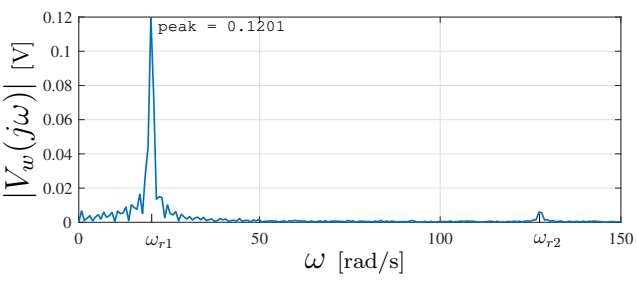

(a)

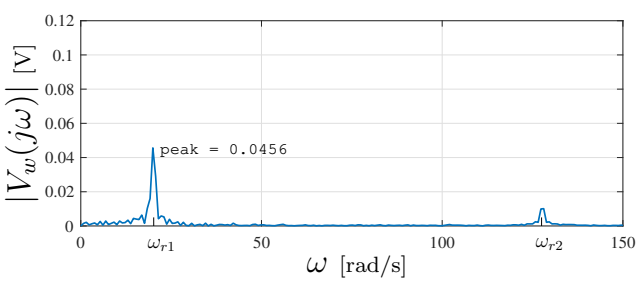

(c)

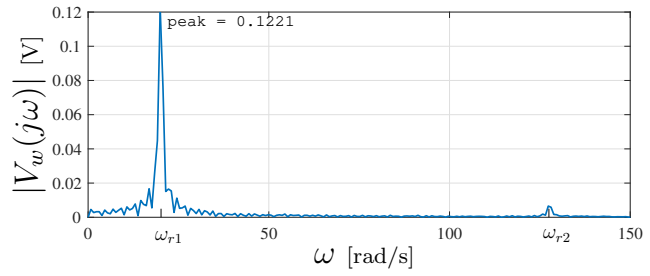

(b)

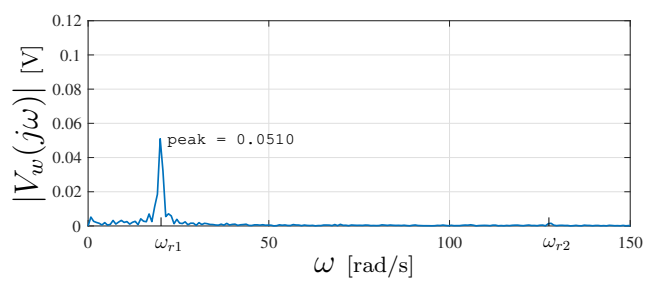

(d)

Figure 23: Magnitude frequency spectrum of the strain gauge output signals $V_{w}(t)$ shown in Fig. 22: no filters (a), derivatives limiter (b), ZVD Input shaper (c), rectangular smoothers (d).

ities cause large tracking errors of the actuator and mechanical noise.

As above mentioned, ZVD input shaper and rectangular smoothers produce a noticeable reduction of the residual vibration at the end of motion but do not completely suppress it as expected. The cause is probably the dynamics of the motor which is not able to exactly track the desired reference motion obtained by filtering the signal composed by ramp functions. This is proved by the results shown in Fig. 24, where the level of residual vibration measured in the experimental tests is compared with the level of vibration obtained on the nominal model of the flexible beam by considering as input the actual motor position and the desired reference position. If the actual motor position is considered the simulated results (reported with a solid red line) resemble very closely the experimental results, and the residual vibration at the end of motion remains. If the reference signal filtered by ZVD 


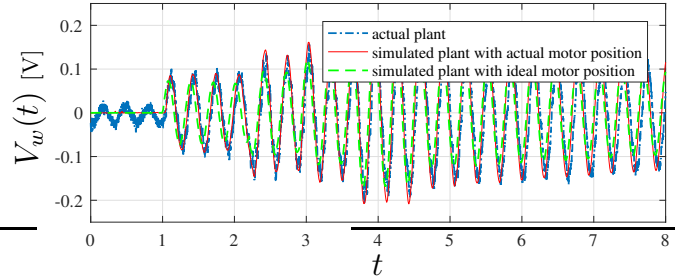

(a)

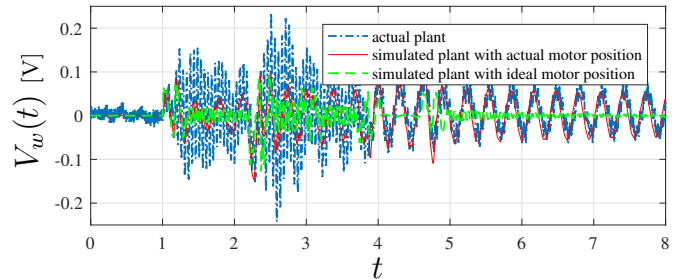

(c)

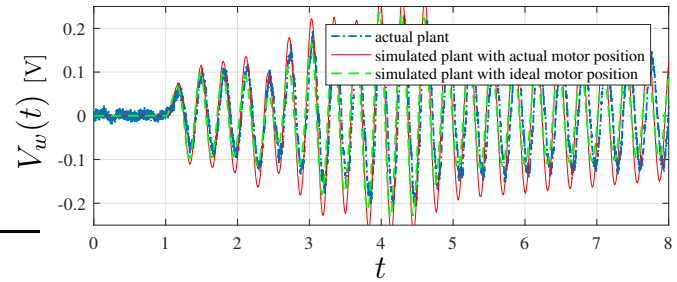

(b)

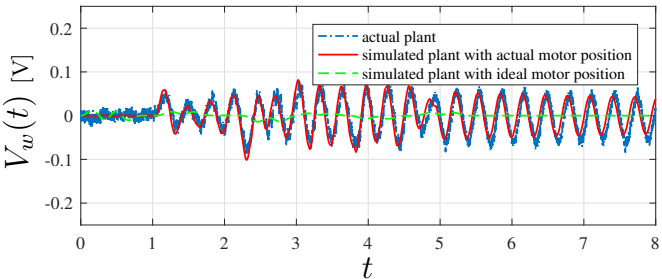

(d)

Figure 24: Comparison between the vibrations measured on the experimental setup and the vibrations induced on the nominal model of flexible beam by the ideal motion obtained by filtering a signal composed by ramp functions: no filters (a), derivatives limiter (b), ZVD Input shaper (c), rectangular smoothers (d).

Input shaper and by the two rectangular smoother is directly provided to the nominal model of the plant (see the dashed green line in Fig. 24(c) and (d)) the residual vibrations vanish as soon as the motion stops (in the case of ZVD Input Shaper a small residual vibration tied to $\omega_{r 2}$ is still present, while the low-pass characteristics of the rectangular smoother leads to a complete vibration suppression).

\subsection{Sawtooth reference input}

The reference signal (24) is defined by $v=0.03 \mathrm{~m} / \mathrm{s}$ and $\tau=2.5 \mathrm{~s}$. In order to comply with the given kinematic constraints, on the basis of the equations (30), the parameters of the rectangular smoothers have been 




(a)

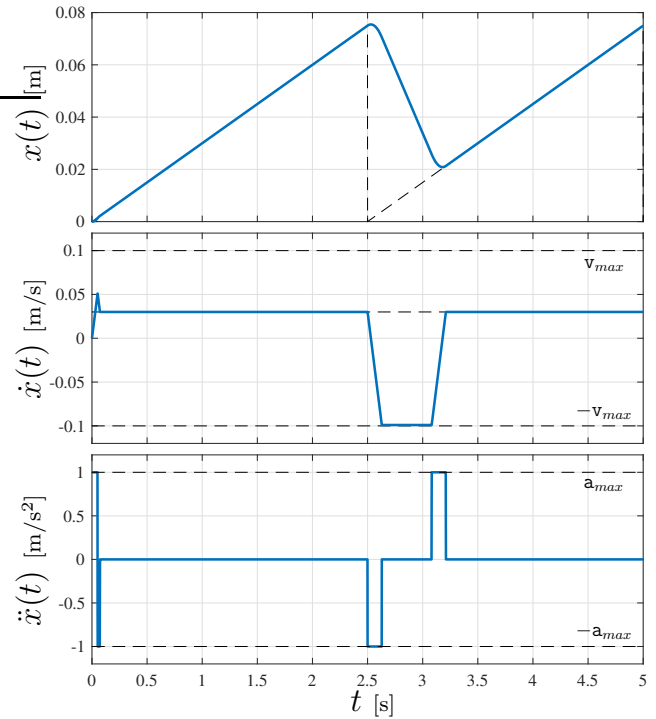

(b)

Figure 25: Position, velocity and acceleration profiles of the sawtooth reference input, filtered by a cascade of two rectangular smoothers (a) and by a second-order derivatives limiter (b), used in the experiments.

selected as

$$
k=2 \Leftrightarrow T=0.6228 \mathrm{~s}, T_{1}=0.1204 \mathrm{~s} .
$$

With this value of $T$, the actual maximum velocity is $\mathrm{v}_{\max }^{\star}=0.0904 \mathrm{~m} / \mathrm{s}$, as shown in Fig. 25(a) where the position, velocity and acceleration profiles of the smoothed sawtooth signal are reported. In the same figure, the profiles obtained with the derivatives limiter are shown. It is worth noticing that in the two cases these profiles are very similar. Accordingly, with this type of reference input and with the given limits, the performances of the smoothers and of the derivatives limiter in terms of vibrations level are similar, see Fig. 26(b) and (d). The FFT analysis performed on $V_{w}(t)$, by considering several repetitions of reference trajectory, confirms that, with re- 


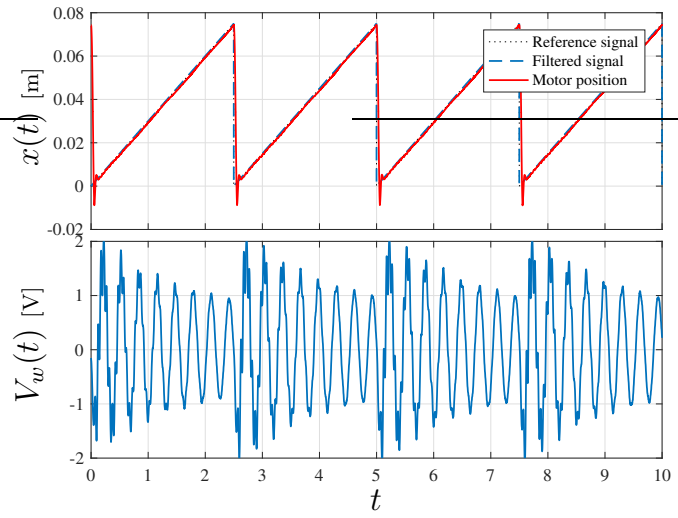

(a)
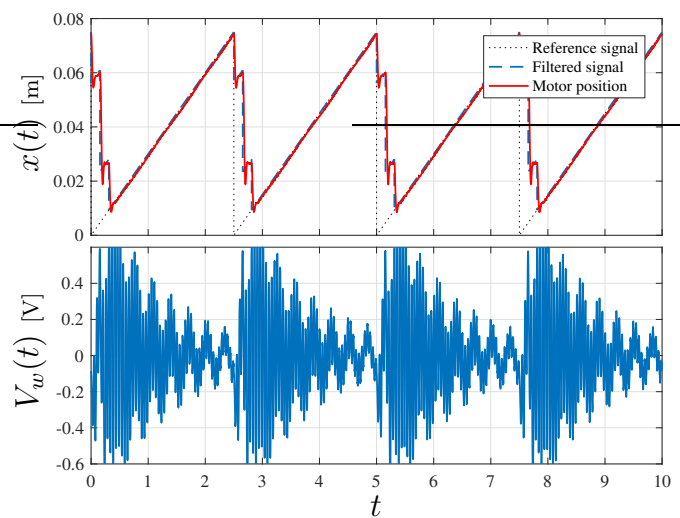

(c)

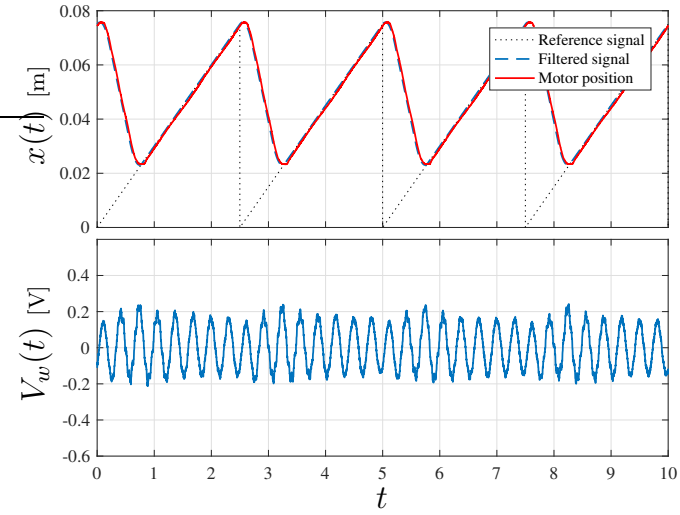

(b)

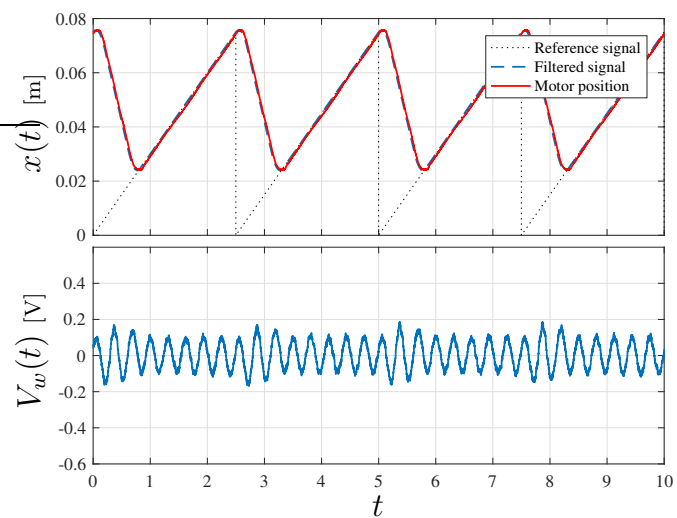

(d)

Figure 26: Reference trajectories obtained by filtering a sawtooth signal with different methods and vibrations induced on the flexible beam: no filters (a), derivatives limiter (b), ZVD Input shaper (c), rectangular smoothers (d). 


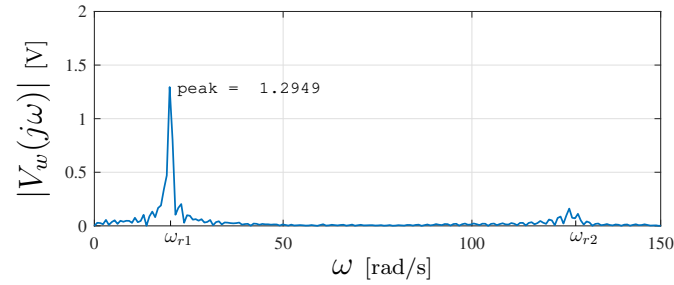

(a)

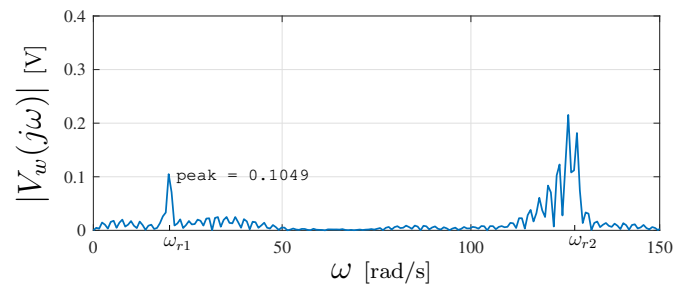

(c)

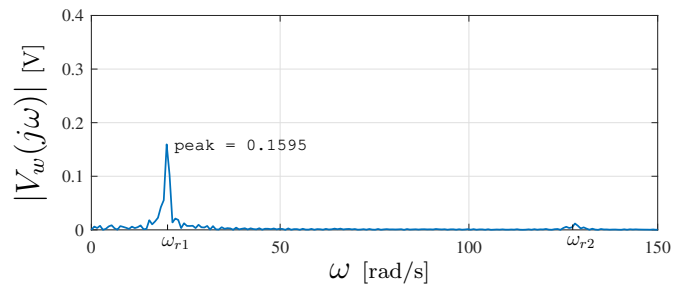

(b)

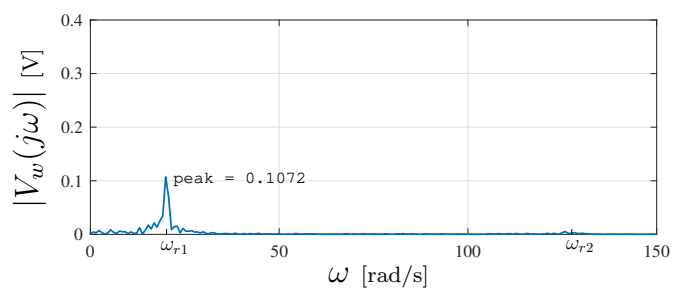

(d)

Figure 27: Magnitude frequency spectrum of the strain gauge output signals $V_{w}(t)$ shown in Fig. 26: no filters (a), derivatives limiter (b), ZVD Input shaper (c), rectangular smoothers (d).

spect to the unfiltered signal, the derivatives limiter produces a reduction of the first vibratory mode of $87.68 \%$, while the rectangular smoothers guarantee $-91.72 \%$. A similar results $(-91.90 \%)$ is obtained with the application of the ZVD input shaper, but again the discontinuities on the position profile, caused by the phase delay compensator, induce large oscillations at frequencies close to $\omega_{r 2}$ as highlighted by Fig. 26(c) in the time domain and by Fig. 27(c) in the frequency domain.

As for the signal composed by ramp functions, also in the case of the sawtooth signal the simulative (nominal) model provides results very similar to the experimental tests (except for the large variations occurring without any filtering action, that in the real case do not seem subject to superposition principle), see Fig. 28. When the actual motor position is applied to the simulated model the level of residual vibration is comparable with the 


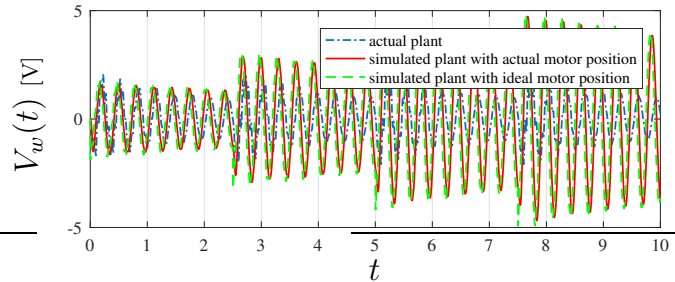

(a)

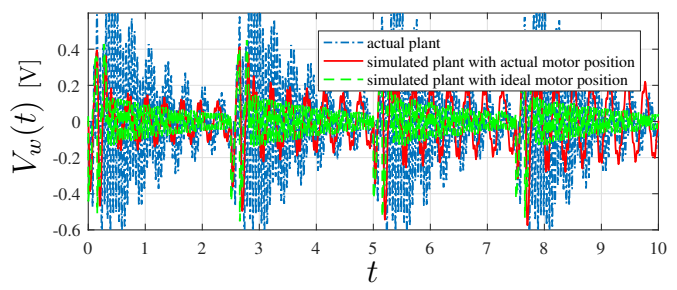

(c)

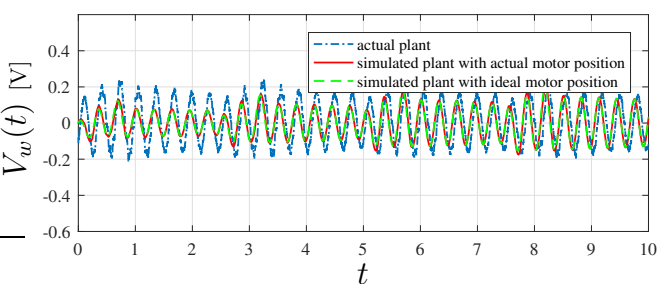

(b)

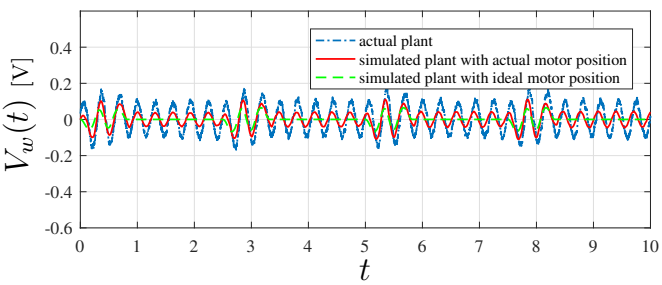

(d)

Figure 28: Comparison between the vibrations measured on the experimental setup and the vibrations induced on the nominal model of flexible beam by the ideal motion obtained by filtering the sawtooth reference input: no filters (a), derivatives limiter (b), ZVD Input shaper (c), rectangular smoothers $(d)$.

measurements obtained in the experimental tests, but if the ideal reference motion is considered, the two rectangular smoothers guarantee a complete suppression of the vibration as shown in Fig. 28(d). In the case, of the ZVD Input shaper only the vibration caused by the resonant mode at $\omega_{r 2}$ remains, see Fig. 28(c). As already mentioned, the reason of the discrepancy between experiments and simulations is ascribable to the motor which cannot perfectly track the reference signal.

\section{Conclusions}

A feed-forward technique based on exponential and rectangular smoothers is proposed to achieve asymptotic perfect tracking of constant velocity signals in systems characterized by vibratory modes of the plant and kinematic 
constraints of the actuation. The closed form expression of the smoothers parameters that guarantees these objectives have been deduced. The scheme has been implemented experimentally and compared to existing techniques in the literature, proving its effectiveness when vibration reduction and bounded derivatives are required in applications based on precise tracking of constant velocity signals.

\section{Appendix A. Exponential smoothers discretization}

The discrete-time expression of the exponential smoother, obtained by Z-transforming the expression of $F_{\text {exp }}(s)$ in (5) with a zero-order hold, is

$$
F_{e x p}(z)=\frac{1-e^{\sigma T_{s}}}{1-e^{\sigma N T_{s}}} \frac{\left(1-e^{\sigma N T_{s}} z^{-N}\right) z^{-1}}{1-e^{\sigma T_{s}} z^{-1}}
$$

where $N=\operatorname{round}\left(T / T_{s}\right)$, being $T_{s}$ the sampling time. Since the scheme of the exponential smoother reported in Fig. 5, which also provides the first derivative of the filtered signal, cannot directly translated in the discrete-time domain, it is necessary to find the expression of a second filter performing the derivative operation. This discrete-time filter can be obtained by Ztransforming $F_{\text {exp der }}(s)=s F_{\text {exp }}(s)$, i.e.

$$
F_{e x p, d e r}(z)=\frac{-\sigma\left(1-e^{\left.\sigma T_{s}\right)}\right.}{1-e^{\sigma N T_{s}}} \frac{\left(1-e^{\sigma N T_{s}} z^{-N}\right)\left(1-z^{-1}\right)}{1-e^{\sigma T_{s}} z^{-1}} .
$$

From the combination of the two filters (A.1) and (A.2) the scheme of Fig. A.29 is deduced.

In case $\sigma=0$ the filter becomes a standard rectangular smoother and its discrete-time expression assumes the simple form reported in Fig. A.30. 




Figure A.29: Discrete-time exponential smoother with derivative computation.

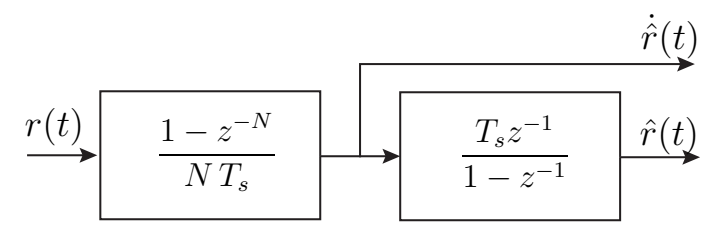

Figure A.30: Discrete-time rectangular smoother with derivative computation.

\section{References}

Béarée, R., 2014. New damped-jerk trajectory for vibration reduction. Control Engineering Practice 28, 112 - 120. doi:http://dx.doi.org/10. 1016/j . conengprac. 2014.03.010.

Bellezza, F., Lanari, L., Ulivi, G., 1990. Exact modeling of the flexible slewing link, in: Proceedings., IEEE International Conference on Robotics and Automation, pp. 734-739 vol.1. doi:10.1109/ROBOT.1990.126073.

Besset, P., Béarée, R., 2017. Fir filter-based online jerk-constrained trajectory generation. Control Engineering Practice 66, 169 - 180. doi:https: //doi.org/10.1016/j. conengprac.2017.06.015.

Biagiotti, L., Melchiorri, C., 2012. FIR filters for online trajectory planning with time- and frequency-domain specifications. Control Engineering Prac- 
tice 20, 1385 - 1399. doi:http://dx.doi.org/10.1016/j.conengprac. 2012.08 .005 .

Biagiotti, L., Melchiorri, C., Moriello, L., 2016. Optimal trajectories for vibration reduction based on exponential filters. IEEE Transactions on Control Systems Technology 24, 609-622. doi:10.1109/TCST . 2015. 2460693.

Biagiotti, L., Zanasi, R., 2010. Time-optimal regulation of a chain of integrators with saturated input and internal variables: an application to trajectory planning, in: NOLCOS 2010, 8th IFAC Symposium on Nonlinear Control Systems, Bologna.

Croft, D., Devasia, S., 1999. Vibration compensation for high speed scanning tunneling microscopy. Review of Scientific Instruments 70.

Ezair, B., Tassa, T., Shiller, Z., . Planning high order trajectories with general initial and final conditions and asymmetric bounds. To appear in International Journal of Robotics Research .

Gerelli, O., Bianco, C., 2010. A discrete-time filter for the on-line generation of trajectories with bounded velocity, acceleration, and jerk, in: Robotics and Automation (ICRA), 2010 IEEE International Conference on, pp. 3989 -3994. doi:10.1109/ROBOT. 2010.5509712.

Guarino Lo Bianco, C., Ghilardelli, F., 2014. A discrete-time filter for the generation of signals with asymmetric and variable bounds on velocity, acceleration, and jerk. Industrial Electronics, IEEE Transactions on 61, 4115-4125. doi:10.1109/TIE. 2013.2284135. 
Haschke, R., Weitnauer, E., Ritter, H., 2008. On-line planning of timeoptimal, jerk-limited trajectories, in: IROS, pp. 3248-3253.

Kamel, A., Lange, F., Hirzinger, G., 2008. New aspects of input shaping control to damp oscillations of a compliant force sensor, in: 2008 IEEE Int. Conf. on Robotics and Automation, pp. 2629-2635. doi:10.1109/ ROBOT . 2008.4543609.

Kane, T.R., Ryan, R., Banerjee, A.K., 1987. Dynamics of a cantilever beam attached to a moving base. Journal of Guidance, Control, and Dynamics 10, 139-151. doi:10.2514/3.20195.

Kröger, T., Tomiczek, A., Wahl, F.M., 2006. Towards on-line trajectory computation, in: Proc. of the IEEE/RSJ Int. Conf. on Intelligent Robots and Systems, Beijing, China. pp. 736-741.

Kröger, T., Wahl, F.M., 2010. On-line trajectory generation: Basic concepts for instantaneous reactions to unforeseen events. IEEE Trans. on Robotics 26, 94-111.

LinMot, 2019. Linmot homepage. URL: www.linmot.com.

Lo Bianco, C.G., Tonielli, A., Zanasi, R., 2000. Nonlinear filters for the generation of smooth trajectories. Automatica 36, 439-448.

Luo, Z.H., Kitamura, N., Guo, B.Z., 1995. Shear force feedback control of flexible robot arms. IEEE Transactions on Robotics and Automation 11, 760-765. doi:10.1109/70.466607. 
Masterson, R., Singhose, W., Seering, W., 1998. Input shaping for constantvelocity scanning with flexible sensors.

Meckl, P., Arestides, P., 1998. Optimized s-curve motion profiles for minimum residual vibration, in: Proceedings of the American Control Conference, Philadelphia, Pennsylvania. pp. 2627-2631.

Muenchhof, M., Singh, T., 2003. Jerk limited time optimal control of flexible structures. Journal of Dynamic Systems, Measurement, and Control 125, 139-142. URL: http://dx.doi.org/10.1115/1.1543552, doi:10.1115/ 1.1543552 .

Nakabayashi, Y., Okajima, H., Matsunaga, N., 2017. Signal limitation filter to satisfy velocity and acceleration constraints for arbitrary input signals, in: 2017 56th Annual Conference of the Society of Instrument and Control Engineers of Japan (SICE), pp. 1197-1201. doi:10.23919/SICE. 2017. 8105481.

Nguyen, K.D., Chen, I.m., Ng, T.c., 2007. Planning algorithms for s-curve trajectories. 2007 IEEE/ASME international conference on advanced intelligent mechatronics 1, 1-6. doi:10.1109/AIM. 2007.4412440.

Peng, D.W., Singh, T., Milano, M., 2015. Zero-phase velocity tracking of vibratory systems. Control Engineering Practice 40, 93 - 101. doi:https: //doi.org/10.1016/j. conengprac.2015.03.008.

Piazzi, A., Visioli, A., 2000. Minimum-time system-inversion-based motion planning for residual vibration reduction. Mechatronics, IEEE/ASME Transactions on 5, 12-22. doi:10.1109/3516.828585. 
Pilbauer, D., Michiels, W., Vyhlidal, T., 2017. Distributed delay input shaper design by optimizing smooth kernel functions. Journal of the Franklin Institute 354, 5463 - 5485. doi:https://doi .org/10.1016/j.jfranklin. 2017.06 .002 .

Singer, N., Singhose, W., Seering, W., 1999. Comparison of filtering methods for reducing residual vibration. European Journal of Control 5, 208-218.

Singer, N.C., Seering, W.P., 1990. Preshaping command inputs to reduce system vibration. ASME Journal of Dynamic Systems, Measurement, and Control 112, 76-82.

Singh, T., 2004. Jerk limited input shapers. Journal of Dynamic Systems, Measurement, and Control 126, 215-219. doi:10.1115/1.1653808.

Singhose, W., Singer, N., Seering, W., 1995. Comparison of command shaping methods for reducing residual vibration, in: Third European Control Conf, pp. 1126-1131.

Thirachai, S., Komeswarakul, P., Supakchukul, U., Suwatthikul, J., 2010. Trapezoidal velocity trajectory generator with speed override capability, in: ICCAS 2010, pp. 1468-1472. doi:10.1109/ICCAS.2010 .5670142.

Vyhlídal, T., Hromvcík, M., 2015. Parameterization of input shapers with delays of various distribution. Automatica 59, 256-263. doi:10.1016/j . automatica.2015.06.025.

Xie, X., Huang, J., Liang, Z., 2013a. Using continuous function to generate shaped command for vibration reduction. Proceedings of the Institution of 
Mechanical Engineers, Part I: Journal of Systems and Control Engineering $227,523-528$.

Xie, X., Huang, J., Liang, Z., 2013b. Vibration reduction for flexible systems by command smoothing. Mechanical Systems and Signal Processing 39, 461 - 470. doi:https://doi.org/10.1016/j.ymssp.2013.02.021. 